


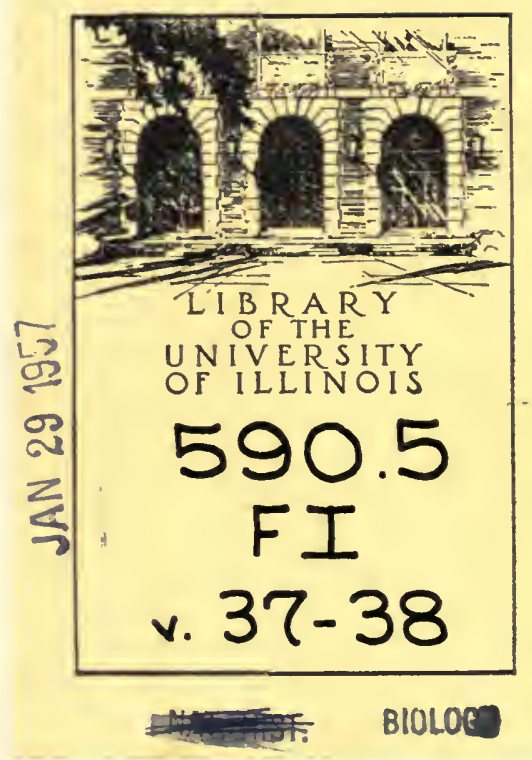


The person charging this material is responsible for its return on or before the Latest Date stamped below.

Theft, mutilation and underlining of books

are reasons for disciplinary action and may

result in dismissal from the University.

UNIVERSITY OF ILLINOIS LIBRARY AT URBANA-CHAMPAIGN

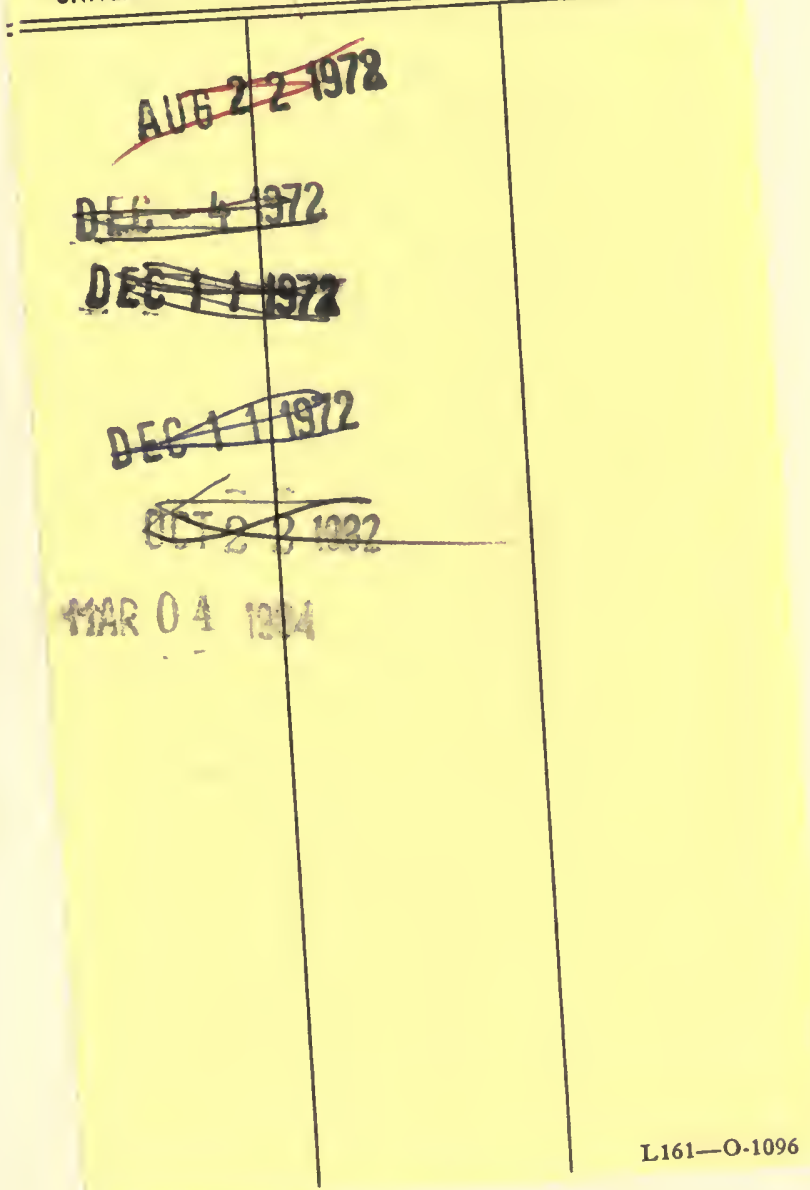







\title{
Bats from Guerrero, Jalisco and Oaxaca, Mexico
}

\author{
LUIS DE LA TORRE
}

\author{
Associate, Division of Mammals
}

This paper is based on a small collection of bats made by the author in Guerrero and Oaxaca from July 22 to August 5, 1952, and in Jalisco from December 7 to 11,1954 . The primary objective of this collecting was to obtain additional specimens of Sturnira to be used for a systematic review of that genus. In sampling caves and other likely habitats, representatives of fourteen species of bats were collected, including the first series to be taken of Enchisthenes harti.

I am indebted to Miss Barbara Lawrence, Museum of Comparative Zoology, and to Dr. E. Raymond Hall, Museum of Natural History, University of Kansas, for the loan of specimens in their care; to Mr. R. W. Hayman, British Museum (Natural History), for comparing specimens of Chilonycteris with the types of $C$. psilotis; and to Mr. and Mrs. Loren P. Woods, Chicago Natural History Museum, for their kindness in making possible my visit to the interesting Jalisco region. The patient and enthusiastic help given by my wife, June, in many capacities is also gratefully acknowledged.

\section{LIST OF SPECIES}

With the exceptions noted, the specimens from Guerrero and Oaxaca were taken in caves. Those from Ciudad Guzmán, Jalisco, were netted at night in a grove of native, small-fruit Ficus trees.

The measurements given are in millimeters and, unless otherwise indicated, apply to the entire series. The average measurement, when given, is followed by the extremes in parentheses.

\section{Balantiopteryx plicata plicata Peters}

Balantiopteryx plicata Peters, 1867, Monatsb. Preuss. Akad. Wiss. Berlin, $1867: 476$. 
Type locality.-Puntarenas, Puntarenas, Costa Rica.

Specimens collected.-Total 32 (2 skins with skulls, 30 in alcohol). Guerrero: $5 \mathrm{mi}$. NW. of Acapulco (12 males, 14 females); $12 \mathrm{mi}$. S. of Mexcala ( 1 female). OAXACA: Tehuantepec ( 5 young females).

Measurements.-Forearm of males from Acapulco 41.3 (40.043.0), females 42.6 (40.0-46.0); female from Mexcala 41.8.

\section{Chilonycteris rubiginosa mexicana Miller}

Chilonycteris mexicana Miller, 1902, Proc. Acad. Nat. Sci. Philadelphia, 54: 401.

C[hilonycteris] $p$ [arnellii] mexicana, Koopman, 1955, Jour. Mamm., 36: 112.

Type locality.-San Blas, Nayarít, Mexico.

Specimens collected.-Total 8 (2 skins with skulls, 6 in alcohol). GUerrero: 5 mi. NW. of Acapulco ( 7 males, 1 female).

Measurements. - Forearm of males 57.3 (55.7-58.8), female 59.5.

Remarks.-It cannot be established that parnelli Gray 1843 has priority over rubiginosa Wagner 1843. The issue of the Proceedings of the Zoological Society of London where the name parnelli first appears is dated April 11, 1843, as shown by Koopman (Jour. Mamm., 1955, 36: 111), but the actual date of publication is October of that year (Duncan, Proc. Zool. Soc. London, 1937: 79). The name rubiginosa appears in the Archiv für Naturgeschichte after a paper read in August, but the exact date of publication is not known. In the absence of conclusive evidence, the better known and more widely used name rubiginosa should be retained.

\section{Chilonycteris psilotis Dobson}

Chilonycteris psilotis Dobson, 1878, Cat. Chirop. Brit. Mus., p. 451. Miller, 1902, Proc. Biol. Soc. Washington, 15: 249-Isthmus of Tehuantepec, Mexico.

Chilonycteris personata, J. A. Allen (not Wagner), 1911, Bull. Amer. Mus. Nat. Hist., $30: 263$. G. M. Allen, 1935, Jour. Mamm., 16: 227. Dalquest and Hall, 1949, Jour. Mamm., 30: 425. Davis and Russell, 1952, Jour. Mamm., 33: 234; 1954, op. cit., 35: 67.

Chilonycteris torrei continentis Sanborn, 1938, Occ.' Pap. Mus. Zool., Univ. Michigan, no. 373: 1-Laguna de Zotz, Petén, Guatemala (type locality).

Type locality.-Here designated as Tehuantepec, Oaxaca, Mexico.

Specimens collected.-Total 3 (2 skins with skulls, 1 in alcohol). Guerrero: $5 \mathrm{mi}$. NW. of Acapulco (3 males).

Measurements.-Forearm 42.5, 43.2, 44.0. 
Remarks.-These specimens agree with the type of C.torrei continentis Sanborn, and with Guatemala specimens that are identical with the types of psilotis. Additional representatives of psilotis have been examined from Mexico, Central America, and Venezuela. Inasmuch as it has not been demonstrated that $C$. psilotis and the Brazilian C. personata are conspecific, the name psilotis, rather than personata, should be used for the small, mainland Chilonycteris.

\section{Pteronotus davyi fulvus Thomas}

Chilonycteris davyi fulvus Thomas, 1892, Ann. Mag. Nat. Hist., (6), 10: 410. Type locality.-Las Peñas, Jalisco, Mexico.

Specimens collected.-Total 11 (in alcohol). Guerrero: $5 \mathrm{mi}$. NW. of Acapulco (9 males, 2 females).

Measurements.-Forearm, males 44.1 (43.2-45.5), females 44.0, 45.0 .

\section{Mormoops megalophylla megalophylla Peters}

Mormops megalophylla Peters. 1864, Monatsb. Preuss. Akad. Wiss. Berlin, 1864: 381 .

Type locality.-Southern Mexico.

Specimens collected.-Total 27 (in alcohol). GUERRERo: $5 \mathrm{mi}$. NW. of Acapulco (27 males, no females found).

Measurements.-Forearm 54.9 (53.0-59.0).

\section{Micronycteris megalotis mexicana Miller}

Micronycteris megalotis mexicana Miller, 1898, Proc. Acad. Nat. Sci. Philadelphia, 50 : 329.

Type locality.-15 mi. S. of Ciudad Guzmán, Platanar, Jalisco, Mexico.

Specimens collected.-1 (in alcohol). JALISco: $2 \mathrm{mi}$. N. of Ciudad Guzmán (1 female).

Measurements.-Forearm 36.3.

\section{Glossophaga soricina leachi Gray}

Monophyllus leachii Gray, 1844, Voyage of the Sulphur, Zool., 1: 18.

Type locality.-Realejo, Nicaragua.

Specimens collected.-Total 9 (in alcohol). Guerrero: $5 \mathrm{mi}$. NW. of Acapulco (6 males, 3 females).

Measurements.-Forearm 36.3 (35.0-37.5). 
Remarks.--One specimen was taken in a mouse trap baited with ripe banana.

\section{Leptonycteris nivalis nivalis Saussure}

$M[=I$ schnoglossa $]$ nivalis Saussure, 1860, Rev. Mag. Zool., Paris (2), 12: 492.

Leptonycteris nivalis yerbabuenae Martinez and Villa, 1940, An. Inst. Biol.

Univ. Nac. México, 11: 313-Yerbabuena, Guerrero, Mexico (type locality).

Type locality.-Near snow line on Mount Orizaba, Veracruz, Mexico.

Specimens collected.-1 (in alcohol). GUERRERo: $12 \mathrm{mi}$. S. of Mexcala (1 male).

Measurements.-Forearm 54.0.

Remarks.-This specimen, a topotype of yerbabuenae, is indistinguishable from a topotype of true nivalis from Orizaba. In size, it also agrees with specimens from Colima, but is smaller than those from Nuevo León, Texas, and Arizona. A size cline from North to South is indicated.

\section{Artibeus jamaicensis jamaicensis Leach}

Artibeus jamaicensis Leach, 1821, Trans. Linn. Soc. London, 13: 75.

Type locality.--Jamaica.

Specimens collected.-Total 5 (in alcohol). JALISCO: $2 \mathrm{mi}$. N. of Ciudad Guzmán (3 males, 2 females).

Measurements.-Forearm 58.0 (55.5-60.0).

Remarks.-All specimens in the series have the third upper molar.

\section{Artibeus cinereus toltecus Saussure}

Stenoderma tolteca Saussure, 1860, Rev. Mag. Zool., Paris, (2), 12: 427.

Type locality.-Mirador, Veracruz, Mexico.

Specimens collected.--Total 8 (in alcohol). JALISco: 2 mi. N. of Ciudad Guzmán ( 5 males, 3 females).

Measurements.-Forearm 40.0 (37.0-42.0).

Remarks.-The palate in two individuals appeared abnormally wide, and the arrangement of the upper teeth (pl. 30, fig. 1) differed significantly from the normal condition in Artibeus cinereus (pl. 30, figs. $4,5,6$ ). Study of the skulls revealed that they were of extreme age, and the labial edge of the maxillary bone had been eroded (pl. 30, fig. 2) leaving a good part of the roots unprotected. It is probable that if this weakened condition develops early in life, the 


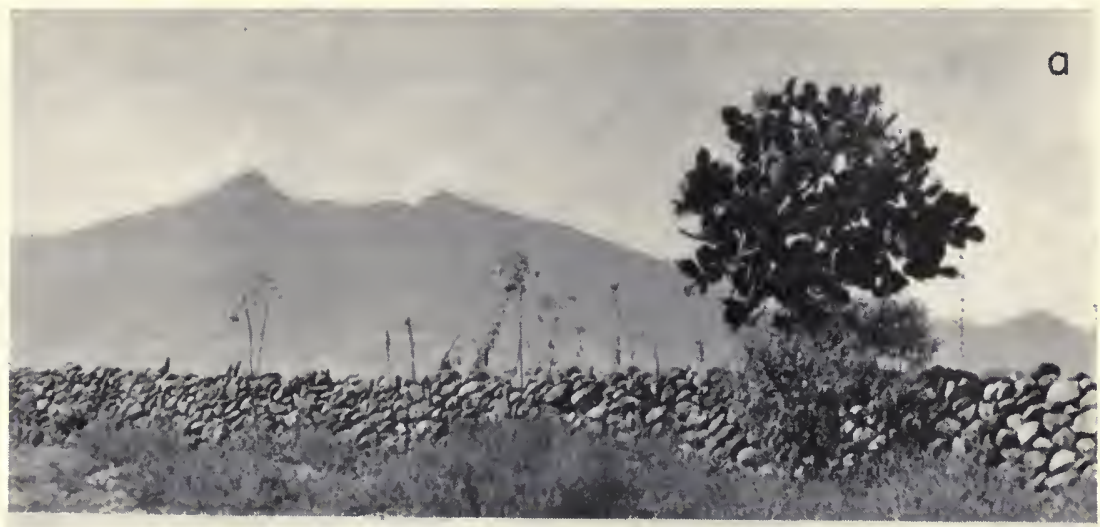

b
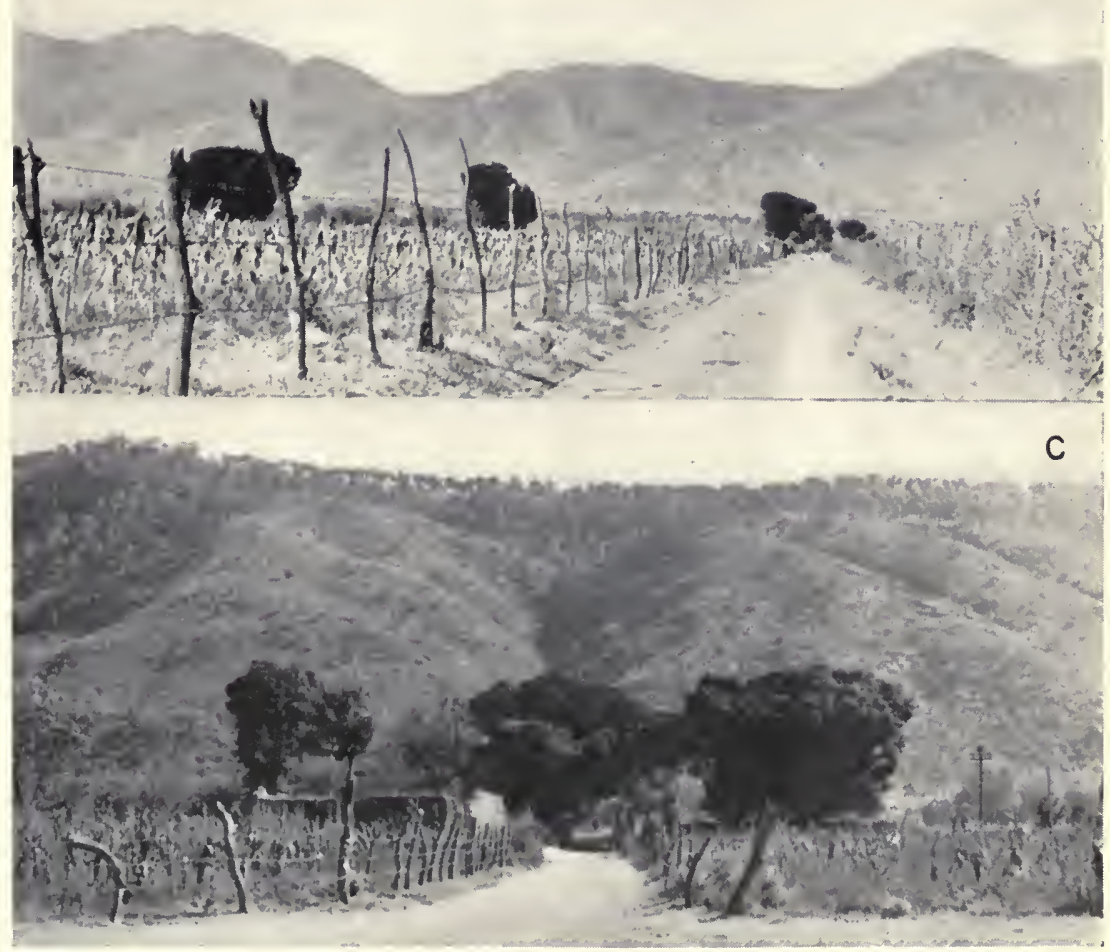

Fig. 146. $a$, View from the Ciudad Guzmán basin to the southwest. Volcán de Colima in the distance. $b$, View of basin showing scattered fig trees frequented by fruit-eating bats. $c$, Eastern edge of basin showing feeding habitat of Enchisthenes harti, Artibeus j. jamaicensis, A. cinereus toltecus, Sturnira lilium parvidens, and Micronycteris megalotis mexicana. 
lateral mastication movements very likely would alter the alignment of the teeth and cause them to flare out as in this case (pl. 30, fig. 3).

\section{Enchisthenes harti Thomas. Plate 31.}

Artibeus harti Thomas, 1892, Ann. Mag. Nat. Hist., (6), 10: 408.

Enchisthenes harti, Sanborn, 1932, Ann. Carnegie Mus., 21 : 179-Venezuela (skin and skull); Goodwin, 1940, Amer. Mus. Nov., no. 1075: 2-Honduras (skin and skull); Ecuador (incomplete skulls from owl pellets); Goodwin, 1954, Amer. Mus. Nov., no. 1689: 5-Tamaulipas, Mexico (incomplete skull).

Type locality.-Trinidad, British West Indies.

Specimens collected.-Total 12 (1 skin with skull, 11 in alcohol). JALiSCO: 2 mi. $\mathrm{N}$. of Ciudad Guzmán (8 males, 4 females).

Measurements.-Forearm 39.8 (38.2-43.1); greatest length of skull, of a male and two females 20.4, 20.9, 20.9; condylobasal length $18.8,19.0,19.0$; zygomatic breadth 12.1, 12.2, 12.1; mastoid breadth $10.8,10.7,10.6$; palatal length $9.0,9.8,9.1$; postorbital constriction 5.4, 5.6, 5.3; maxillary tooth row 6.8, 7.0, 6.9; greatest width across molars 8.4, 8.6, 8.3; length of mandible 13.1, 13.4, 13.9; lower canine to condyle 12.9, 13.2, 13.5; mandibular tooth row $7.3,7.5,7.3$.

Remarks. -E. harti, previously known from only three complete specimens, was common in the Ficus groves (fig. 146, c) near Ciudad Guzmán. This village lies in a basin rimmed by the Sierra Nevada de Colima on the west (fig. 146, b), and by lower, pine covered hills on the east (fig. 146, c).

The feeding habits of Enchisthenes, judging from the individuals observed, probably make it a rather easy prey of owls. The fruit eaten in this area is small, about a centimeter in diameter. It is quickly snipped from the tree in flight, and carried to a convenient branch where it is eaten. The forearms are used to handle and turn the fruit as parts of it are consumed, and it is in these movements that the exposed bat probably is seen by its predators.

It is interesting to note that $E$. harti and Sturnira lilium parvidens, both tropical species, were taken on one occasion when the temperature was only $36^{\circ} \mathrm{F}$.

\section{Desmodus rotundus murinus Wagner}

$D$ [esmodus] murinus Wagner, 1840, Schreber's Saugth., Suppl., 1: 377.

Type locality.-Mexico. 
Specimens collected.-Total 2 (in alcohol). GUERrero: 5 mi. NW. of Acapulco (1 male, 1 female).

Measurements.--Forearm, male 58.2, female 60.6 .

\section{Molossus sinaloae Allen}

Molossus sinaloae Allen, 1906, Bull. Amer. Mus. Nat. Hist., 22: 236.

Type locality.-Esquinapa, Sinaloa, Mexico.

Specimens collected.-Total 9 females (in alcohol). GUERrERo: $15 \mathrm{mi}$. NW. of Acapulco.

Measurements.-Forearm 47.9 (46.5-49.8).

Remarks.-These specimens were taken under the dry fronds of a coconut palm.

Miller and Kellogg (1955, U. S. Nat. Mus. Bull. 205: 119) follow G. M. Allen in regarding sinaloae a race of rufus Geoffroy. $M$. sinaloae, however, is a medium size species and is distinct from the larger $M$. rufus of which nigricans Miller is the northern race. $M$. r. nigricans and $M . r$. rufus differ only in average size, while $M$. sinaloae can always be separated from $M$. rufus on absolute characters. 


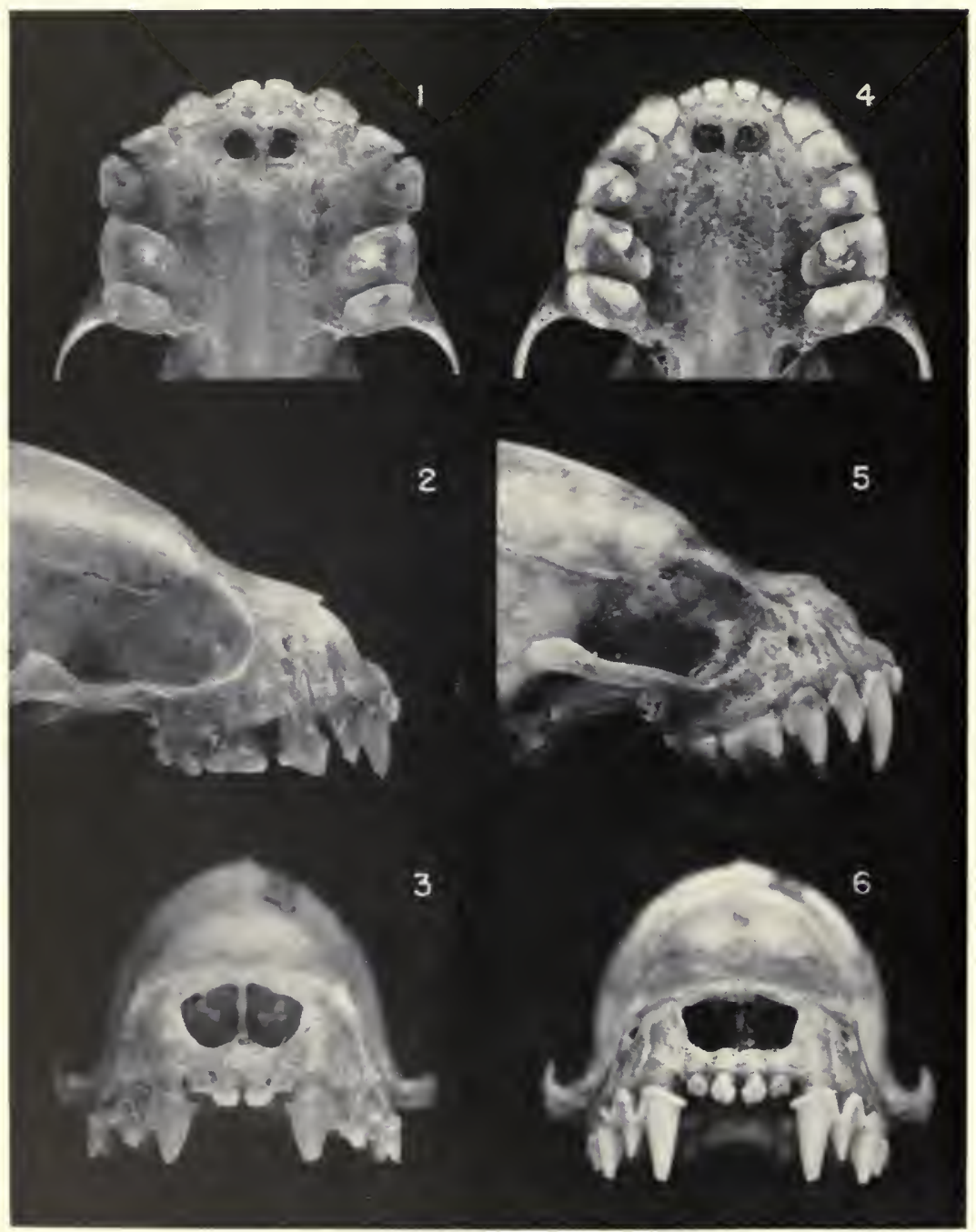

Palate, maxilla and rostrum of Artibeus cinereus toltecus: 1-3, distorted condition in female of extreme age; $4-6$, normal condition in adult female. $\times 4$. 

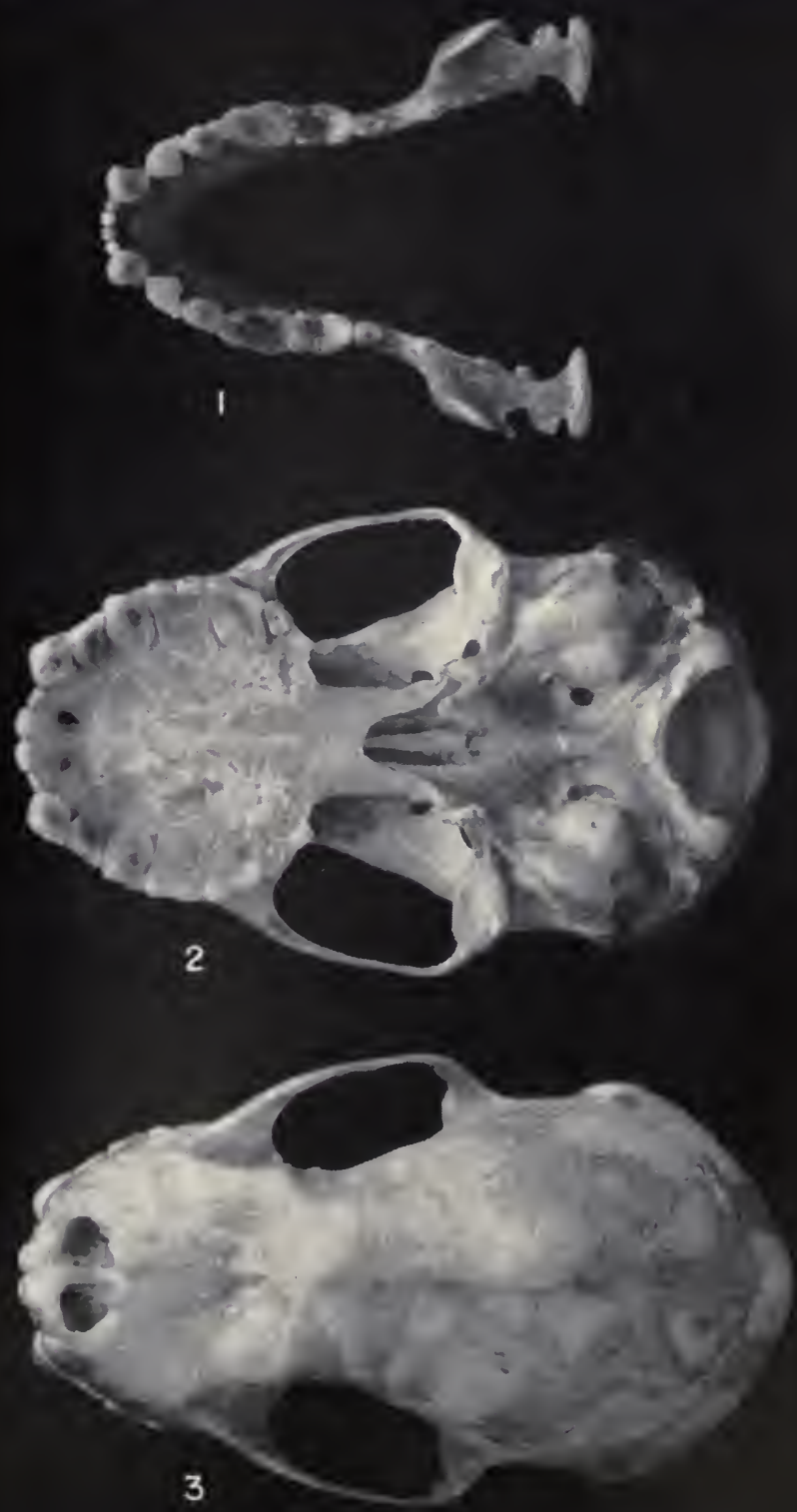

Enchisthenes harti: 1, mandible; 2, ventral surface of skull; 3, dorsal surface of skull. $\times 4$. 



\section{Index}

Principal discussion and all new names indicated by bold-faced type.

Abo Formation 389

Abraeinae 605, 610

Abraeus 601

acicularis 620

phyllobius 601

punctiger 624

vividulus 601

abruptum, Platysoma 629

aburri, Aburria 21

Aburria aburri 21

acanthognathus, Lophodolus 299

Acanthotermes 468, 486, 512

Achalinus spinalis 174

acicularis, Abraeus 620

Bacanius 605, 606, 620

Aciculitermes 515

acme, Bulimulus 325

Acorhinotermes 469, 493, 509

Acritus 602, 605

biloculatus 612

caledoniae $605,607,612,614$

lilliputianus 612

schmidti $605,607,612,614$

actinopterygians 388

Actitis macularia $\mathbf{1 5 4}$

aculeatus, Argyropelecus 275

acuminata, Erolia 154

acunhae, Nesospiza 147, 152

horny palate 151

acutirostris, Calamaria 199

Adioryx 126

Holocentrus 92, 109

Admiral Formation 389

adusta, Nenia 305

tumens, Nenia 305

aegyptiaca, Tadarida 595

aegyptiacus, Rousettus 595

Aeletes 620

crenatus $605,606,610$

daubani 610

laevis 610

longipes 614

politus 610

aequatorialis, Penelope 17

aequinoctialis, Succinea 305

Afrosubulitermes $\mathbf{5 1 5}$

agilis, Mus 648

Agouti paca 215

Ahamitermes 469, 503, 510

Ailuropoda 44

\author{
Aistopoda 531 \\ Akodon aerosus 234 \\ berlepschii 231 \\ jelskii 254 \\ pulcherrimus 227 \\ pyrrhotis 228 \\ nigrita 237
}

akodoni, Amblyopinus 228

alatus, Lampanyctus 289

alba, Egretta 153

Monopterus 53, 59

Muraena 59

alberti, Crax 17

albinism, in cracids 19

albiventer, Calamaria 199

albopunctata, Calamaria 199

alidae, Calamaria 200

Allodontermes 473, 513

Allognathotermes 511

alstoni, Neotomodon 547

alticolus, Edrabius 259

amabilis, Argyropelecus 276

amazonicus, Holochilus 659, 667 brasiliensis 667,669

Ambassis, ecological distribution 81

gymnocephalus $52,54,71$

interrupta $52 \mathrm{ff}, 71$

ecological distribution 82

food 71,84

nalua 53, 72

predator of 68

Amblyopininae $211 \mathrm{ff}$

Amblyopinini 221

Amblyopinodes 216, 218, 220, 222, 247

claviger 249

gahani 248

peruvianus 250

piceus 248

similis 251

travassosi 249

Amblyopinus 212, 214, 216-18, 220, 221

akodoni 228

ancashi 232

angustus 247

bequaerti 247

brandesi 227

chilomysi 239

colombiae 231

emarginatus 239

fuegensis 216,237 
henseli 212, 247

hershkovitzi 238

huilae 242

jelskii 212, 224

kalinowskii 243

kofordi 231

longus 234

marmosae 247

monticolus 228

montivagans 240

oryzomysi 240

pacae 244

punae 227

sanborni 238

schmidti 231

spinipennis 234

waterhousei 219,243

amblyurus, Hemirhamphus 67

Zenarchopterus 52ff, 67

food 67,84

americanus, Ursus 25

Amitermes 467, 469, 472, 473, 477, 480, $483,485,488,491,493,501,503$, 510

Amitermitinae 493, 505, 506, 510, 516, 562

amoenus, Neomyripristis 94

Amphibia, apsidospondylous 388

lepospondylous 388

Amphicynodontinae 44

amplus, Peromyscus difficilis 547

Ampullariidae 303

Ampullarius (Ampullarius) bridgesii 304 insularum 304 papyraceus 303

(Limnopomus) crassus 303 nubilus 303

Anabantoidea, ecological distribution 83

Anacanthotermes 473, 482, 509

anale, Scopelarchus 284

Anapleus 601

Anatidae 153

ancashi, Amblyopinus 232

Ancistrotermes 513

andium, Radiodiscus 308

andivaga, Streptaxis 329

Systrophia 308

andoicus, Bulimulus 324

Angelosaurus 395, 398

angouya, Mus 660

Oryzomys 654

angrandi, Bulimulus 315

kalinowskii, Nenia 306

Nenia 306

urubambensis, Nenia 306

Anguilla borneensis 52ff, 58 ecological distribution 82 food 58,84

predator of 62

Angularitermes 469, 495, 497, 514

Angulitermes 471, 485, 501, 512 angustus, Amblyopinus 247

anguya, Holochilus 647

Mus' (Holochilus) 648, 660, 666

Anodontostoma chacunda 52, 53, 55

Anomodontia 387, 398

distribution, geographic 390 geologic 390

Anoplogaster cornuta 292

Anoplogastridae 292

Anoplotermes 472, 484, 491, 493, 500, 510

Anthias maculatus 73

apaporiensis, Caiman sclerops 340

Apicotermes 473, 485, 511

Apilitermes 511

Apobletes 628

appendiculata 627

montrouzieri $605,607,627$

A pocryptes brachypterus 78 rictuosus 80

Apoda 531

Apodes, ecological distribution 83

Aporodon 644

appendiculata, Apobletes 627

apraeocularis, Calamaria 200

arborifera, Linophryne 300

Archotermopsis 468, 470, 482, 507, 509

Arctibeus lineatus 409

Arctotherium 25

arenarius, Miniopterus natalensis 595

Argas 579ff

boueti 580, 594, 598

(Chiropterargas) boueti 580

confusus $579,586,594,598$

vespertilionis 586, 596, 598

Argasidae 579

argenteus, Diretmus 292

argentimaculata, Sciaena 73

argentimaculatus, Lutianus 54, 55, 73 food 84,85

argentinus, Edrabius 258

argus, Chaetodon 75

Scatophagus 52, 54, 55, 75

argyromus, Myripristis 136

Argyropelecus aculeatus 275

amabilis 276

hemigymnus 274

Ariidae, ecological distribution 86

Ariophantidae 308

Arius caelatus 64

venosus 65

arkansas, Catapleura 377

armatus, Neoniphon 94

Armitermes 495, 514

Arroyo Formation 389

fauna $390 \mathrm{ff}$

artensis, Saprinus 605, 608, 609

arthuri-muelleri, Rugitermes 576

Artibeus cinereus toltecus 698

harti 700

jamaicensis jamaicensis 698

vittatus 405 
ascendens, Bulimulus derelictus 317

ascensionis, Holocentrus 93, 100, 106, 122,134

$$
\text { Perca } 100
$$

Asellia tridens tridens 595

assimilis, Carcinops 619

Astronesthes leucopogon 278

Astronesthidae 278

atahualpa, Epiphragmophora 327

atavus, Tretomys 646

ataxus, Bulimulus 320

ater, Lampanyctus 288

atlantica, Evermannella atrata 284

atlanticus, Diogenichthys 286

Tetragonurus 298

Atlantisia 158, 159

rogersi 142

Atractaspis 168

Atractus 168

atractus, Beloholocentrus 95

atrata atlantica, Evermannella 284

atrum, Cyema 291

attenuata, Vinciguerria 273

auritus christiei, Plecotus 595

Reithrodon 646

australasiae, Saprinus 609

Australian region, termite fauna of 470 , $476,508 \mathrm{ff}$

Australitermes 469, 477, 503, 515

Autunian fauna $389 \mathrm{ff}$

Bacanius 602,605

acicularis $605,606,620$

fauveli $605,622,624,625$

hamatus 620

punctiger $605,606,622,624,625$

remingtoni $605,606,622,624$

sulcisternus 620

Bagrus nemurus 65

Balantiopteryx plicata plicata 695

balbo, Evermannella 284

balnearum, Holochilus 656, 661, 665 Holochilus brasiliensis 665

Barbodes sealei 62

Barbus elongatus 62

Bartramia longicauda 154

Basidentitermes 512

Bathophilus metallicus 281

Bathylagidae 284

Bathylagus glacialis 284

bathyphila, Lampadena 288

bathyphilum, Gonostoma 272

bats, ticks of $579 \mathrm{ff}$

Beanea 95

trivittata 95

beani, Serrivomer 291

beccari, Calamaria 200

Belle Plains Formation 389

Beloholocentrus 95 atractus 95

Belone strongylura 66

benjaminsi, Calamaria 200 benoiti, Hygophum 285

Benthodesmus simonyi 298

bequaerti, Amblyopinus 247

berbicensis, Holochilus 661

Holochilus brasiliensis 655, 669

berlepschi, Crypturellus cinereus 15

Beryciformes 121

Betta unimaculata 53, 54, 70

ecological distribution 82,86

food 70,84

bibulbosus, Eustomias 282

bicolor, Calamaria 192, 200

bicornis, Ceratostethus 52, 54fi, 68

Linophryne 300

Neostethus 68

bidentatus, Urotriainus 563, 564

bigelowi, Eustomias 282

bikolana, Vaimosa 79

bikolanus, Pseudogobius 52, 54, 79

biloculatus, Acritus 612

bitaeniatus, Thaumastus 309

bitorques, Calamaria 200

blakfordi, Yarella 272

boa ferox, Stomias 278

boddaerti, Boleophthalmus 55, 80 food 80,85

Gobius 80

Boleophthalmus boddaerti 55, 80

food 80,85

bonapartei, Nothocercus 12

Bonapartia pedialota 269

borneensis, Anguilla 52ff, 58 ecological distribution 82 food 58, 84

Calamaria 177ff, 194, 200

Gastromyzon 64

Neostethus 52, 54ff, 69

Borneo, North, fishes of $47 \mathrm{ff}$

Borneo Zoological Expedition $47 \mathrm{ff}$

boucardi, Crypturellus 16

boueti, Argas 580, 594

boylii levipes, Peromyscus 545

brachygnathus, Rhinolophus clivosus 595

Brachygobius doriae 52, 77

food 77,85

brachypterus, A pocryptes 78

Holocentrus 109

Gobiopterus 55, 78

brachyrhynchus, Rhynchichthys 93

brachyura, Calamaria 192, 200

brachyurus, Doryichthys 55, 67

Syngnathus 67

brandesi, Amblyopinus 227

brasiliensis amazonicus, Holochilus 667

balnearum, Holochilus 665

berbicensis, Holochilus 655, 669

brasiliensis, Holochilus 662

guianae, Holochilus 668

Hesperomys (Holochilus) 666

Holochilomy 662

Holochilus 645, 648, 653, 654, 657, $\mathbf{6 5 8}, 660,661,666$ 
incarum, Holochilus 668

leucogaster, Holochilus 648, 665

Mus 660, 662, 663, 667

nanus, Halochilus 666, 669

vulpinus, Holochilus 661

braueri, Cyclothone 272

Brazil, shells from $303 \mathrm{ff}$

brevibarbatus, Stomias 279

brevidentatus, Serrivomer 291

bridgesii, Ampullarius 304

bridwelli, Spalacotheroides 690

brooki, Calamaria 179

bruegeli, Calamaria 185, 187

brunnescens, Penelope 17

Bubulcus ibis 153

buccinatus, Oryzomys 654, 660

Buchen, Walter 140

buchi, Calamaria 195, 200

buckleyi, Odontophorus gujanensis 21

Bulbitermes 481, 514

Buliminus aegyptiacus 429, 432

chondriformis 419

heptodon 429

septemdentatus 422

sexdentatus 436

Bulimulidae 309,331

Bulimulus acme 325

andoicus 324

angrandi 315

(Ataxus) infundibulum 320 infundibulum umbilicatellus 320 perforatus 320

cereicola 317

culmineus 315

dentritis 319

derelictus 317

elatus 324

extensus 323

(Geoceras) veruculum 320

(Geopyrgus) turritus 336

hamiltoni 325

infundibulum 320 umbilicatellus 320

jocosensis 322

(Lissoacme) cereicola 317 dentritis 319

derelictus ascendens 317

modestus 318

reconditus 320

rudistriatus 318

scutulatus 319

longitudinalis 316

modestus 318

mutabilis 334

nemorensis 316

obeliscus 322

(Peronaeus) acme 325

andoicus 324

elatus 324

extensus 323

hamiltoni 325

pupiformis 325 pyrgidium 321

proteiformis 335

proteus 335

(Protoglyptus) rivasii 336

pupiformis 325

pyrgidium 321

reconditus 320

revinctus 316

rivasii 336

rudistriatus 318

(Scutalus) angrandi 315

culmineus 315

longitudinalis 316

mutabilis 334

nemorensis 316

phaeocheilus 334

proteus 335

revinctus 316

subjussieui 336

tupacii 316

scutulatus 319

(Spirosulcatus) endospira 336

subjussieui 336

tupacii 316

turritus 336

veruculum 320

Bulimus lamelliferus 434

ooularis 422,438

saulcyi 416

septemdentatus 422

bullisi, Holocentrus 91, 113

bungaroides, Calamaria 200

burtoni, Poliospiza 149

horny palate 151

Butis butis 52, 75

gymnopomus 52, 55, 76

food 76,85

butis, Butis 52, 75

Cheilodipterus 75

caelatus, Arius 64

Tachysurus 55, 64

food 85

caeruleomaculatus, Chelon 53, 68

Mugil 68

Caiman sclerops 339 apaporiensis 340

Calamaria $167 \mathrm{ff}$

acutirostris 199

albiventer 199

albopunctata 199

alidae 200

apraeocularis 200

beccari 200

benjaminsi 200

bicolor 192, 200

bitorques 200

borneensis $177 \mathrm{ff}, 194,200$

brachyura 192, 200

brooki 179

bruegeli 185, 187

buchi 195,200 
bungaroides 200

ceramensis 200

check list $199 \mathrm{ff}$

collaris 201

crassa 192, 201

curta 191, 201

dentition $173 \mathrm{ff}$

döderleini 201

doerianense 199, 201

egregia 201

electa 201

elegans 190, 192, 201

everetti 192, 201

food 174

gervaisi 169, 176, 178, 180, 201

iridescens 182

gimletti 196, 199, 201

göringi 202

grabowskyi 178, 189, 202

gracilis 202

gracillima 196, 199, 202

grayi $183,185,188$

griswoldi 178, 183, 202

hollandi 202

hosei 202

indragirica 202

javanica 202

javanica 202

lineata 196, 202

jeudi 202

joloensis 203

klossi 203

lateralis 203

lautensis 178, 191, 203

leucocephala 169,176, 178, 193, 203

leucogaster 169, 176, 179, 203 dentition $172 \mathrm{ff}$

linnaei 171, 195, 203

lowi 178, 196, 199, 203

lumbricoidea 178, 183, 189, 203 griswoldi 183, 202

lumholtzi 203

margaritophora 191, 192, 203

gastropicta 204

margaritophora 203

mearnsi 204

mecheli 204

melanota 204

mindorensis 204

mjobergi 204

muelleri 192, 204

nuchalis 204

occipitalis 204

ornata 204

pavimentata 193, 204

formosana 205

pavimentata 204

pendleburyi 178, 191, 192, 205

pfefferi 205

polillensis 205

prakkei 190, 192, 205

quinquetaeniata 205 raveni 205

rebentischi 205

schlegeli 205

schmidti 174, 178, 197, 205

semiannulata 205

septentrionalis $169,178, \mathbf{1 9 4}, 206$

simalurensis 189,190,192, 206

smithi 206

sondaica 206

specializations of $168 \mathrm{ff}$

stahlknechti 192, 206

suluensis 178, 190, 192, 206

sumatrana 191, 192, 206

tropica 168, 192, 206

ulmeri 206

uniformis 206

ventralis 196,206

vermiformis 176, 178, 183, 185, 207

grayi 188,207

vermiformis 187,207

virgulata 180, 207

zamboangensis 207

calcarifer, Holocentrus 72

Lates 53, 72

food 84

Calcaritermes 472, 490, 492, 498, 508

caledoniae, Acritus 605, 607, 612, 614

caledoniae, Sternaulax 605, 607, 626

calosoma, Macrostomias 279

canaliculatus, Drymaeus 314

canalifrons, Prorhinotermes 488

canellinus, Holochilus 647, 661, 666

Canis familiaris, weights of jaw muscles 42

capensis, Eptesicus 595

Capritermes 469, 477, 478, 480, 486, 488, $501,503,512$

Captorhina 387

Captorhinikos 391

Captorhinoides 391

Captorhinomorpha 386, 399

distribution, geographic 390 geologic 390

Permian evolution 391

Captorhinus 391

caquetae, Ortalis 19

Caranx ignobilis 53, 55, 72

food 73,84

carapace, of Caretta $374 \mathrm{ff}$

of Lepidochelys $374 \mathrm{ff}$

of Procolpochelys 349

Carbondale Formation 523

carce, Ichthyocampus 54, 55, 67 Syngnathus 67

Carcinops assimilis 619

minuta 618

palans 618

pumilio 605, 608, 616, 619

quattuordecimstriata 616

rubripes 618,619

tibialis 617

troglodytes $605,608,618$ 


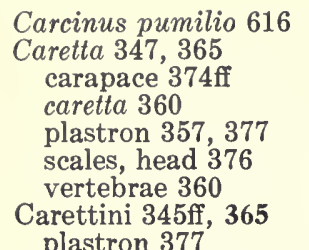

Carollia perspicillata 238, 239

Casea 392

halselli 396

nicholsi 396

Caseidae 387, 392, 399

Caseoides 395, 398

Catapleura arkansas 377

caucae, Crypturellus soui 15 Ortalis 19

Caulolepis longidens 292 subulidens 293

caurinus, Reithrodon 646

cavifrons, Constrictotermes 474

Cavitermes 469, 494, 512

Centrobranchus nigro-ocellatum 286

Cephalaspis 449 novaescotiae 454

Cephalofarer 95

Cephalotermes 469, 484, 493, 495, 510

ceramensis, Calamaria 200

Ceratiidae 299

Ceratostethus bicornis 52, 54ff, 68

Ceratotermes 511

cereicola, Bulimulus 317

Ceylonitermellus 469, 515

Ceylonitermes 481, 497, 514

chacarius, Holochilus 655, 656, 663, 665

chacunda, Anodontostoma 52, 53, 55 Clupanodon 55

Chaerephon major 595

Chaetodon argus 75

Chamaepetes goudotii goudotii 21

Chanda nalua 72

chapalmalense, Proreithrodon 646

chatareus, Coius 75

Toxotes 52, 54, 55, 75

food 75,85

Chauliodontidae 277

Chauliodus danae 277 sloanei 277

chavesi, Lampadena 288

Chelon caeruleomaculatus 53, 68 sp. 54, 68

Chelone grandaeva 345 longiceps 345

Chelonia 366 girundica 366,378 grandaeva 345 gwinneri 364,377 mydas 360,378 neurals 353 sismondai 366,378 vertebrae 360
Cheloniidae, limbs 364, 378 phylogeny $363 \mathrm{ff}$ scales, head 376

Cheloniinae, classification 365

Chelonini 366 plastron 377

Chiasmodon niger pluriradiatus 297

Chiasmodontidae 297

Chiasognathe 499

Chilomys sp. 240, 242

chilomysi, Amblyopinus 239

Chilonycteris davyi fulvus 697 parnelli 696

personata 696

psilotis 696

rubiginosa mexicana 696

torrei continentis 696

chionea, Helicina 305

Chiropterargas 579, 580

Chirostomias pliopterus 281

chocoensis, Ortalis 20

chondriformis, Buliminus 419 Jaminia 419

Pupa 419

Chondrula broti 441

florieni 432, 441

ghilanensis 441

ledereri 441

libanica 423

limbodentata 441

louisi 441

Massei 419

michoni 432

septemdentata 422

tricuspidata 441

triticea 441

Chondrulus heptodon 429

Chondrus l'hotellieri 440 ovularis 438

septemdentatus 422,440

Chondrusa saulcyi impressa 441 septemdentatus 441

Choza Formation 389 fauna $390 \mathrm{ff}$

christiei, Plecotus auritus 595

chrysogaster, Holochilus 661

chrysosoma, Cyprinogobius 52, 77

Lophogobius 77

Cichlherminia 145

cinereiceps, Ortalis 20

cinereus, Crypturellus 15 toltecus, Artibeus 698

Clarias teysmanni $52,54,65$ ecological distribution 82 food 65,84

claromphalos, Epiphragmophora 327

Clausiliidae 305

clausomphalos, Epiphragmophora 327 inferior, Epiphragmophora 327

claviger, Amblyopinodes 249

Clear Fork Group 388ff, 397

fauna of 390 
Climatius 458, 459

clivosus brachygnathus, Rhinolophus 595

Clupanodon chacunda $\mathbf{5 5}$

Clyde Formation 389

Coarctotermes $473,481,486,487,489$, 501,514

cocco, Gonichthys 286

Coilia coomansi 54, 57

Coius chatareus 75

collaris, Calamaria 201

colombiae, Amblyopinus 231

colombiae, Cryptomorpha 572

Colombian Zoological Expedition

(1948-52) 214, 221

map of collecting stations 11

colonization of Tristan da Cunha 159

Coluber 169

columbiana, Ortalis 19

columbianus, Crypturellus boucardi 16

comeri, Porphyriornis 142

commersoni, Stolephorus 52, 56

food 56,84

competition, among termites 474

confusus, Argas 579, 586, 594

connexum, Platysoma 605, 607, 628, 629

Conover, Boardman 9

Constrictotermes 469, 481, 496, 497, 514 cavifrons 474

cyphergaster 474

contiguum, Platysoma 629

Convexitermes 497, 515

coomansi, Coilia 54, 57

Coptotermes 468, 469, 472, 478, 483, 484, $488,493,509$

Coptotermitinae 516

cor, Megaderma 580, 595

cora, Neopetraeus 335

unicolor, Neopetraeus 335

Corica, predator of 80

soborna $52,54,57$

Cornicapritermes 495, 512

Corniger 96, 122

Cornitermes 473, 495, 514

cornuta, Anoplogaster 292

cornutus, Hoplostethus 292

coruscus, Holocentrus 92, 100, 112

Cotylorhynchus $392,397 \mathrm{ff}$

hancocki 396

romeri 396

Cotylosauria 386

couesi, Cryptopsaras 299

crassa, Calamaria 192, 201

crassus, Ampullarius 303

Crax alberti 17

globulosa 16

rubra rubra 17

crenatus, Aeletes 605, 606, 610

Crenetermes 511

Crepititermes 494, 512

cretaceus, Neopetraeus 335

Cricetidae 218

Crithagra 147
Crithagroides 147, 148

crossopterygians 388

Cryptommatus jansoni 260

Cryptomorpha colombiae 572

Cryptopsaras couesi 299

Cryptotermes 472, 478, 487, 490, 492, 508 dudleyi 492

havilandi 487

Crypturellus boucardi columbianus 16 cinereus berlepschi 15

soui caucae 15

undulatus yapura 16

Ctenogobius sp. 55,77

Ctenomys 215, 216, 220

fulvus 259

magellanicus dicki 237

opimus nigriceps 228, 252, 258

peruanus 254, 259

Cubitermes 485, 511

culmineus, Bulimulus 315

cumanensis, Pipile 21

cuniculoides, Reithrodon 646

Cuniculus taczanowskii 215, 244

cuprarius, Lampanyctus 288

curta, Calamaria 191, 201

Curvitermes 468, 495, 514

cyaneocupreus, Saprinus 610

cyaneus, Hister 608

Saprinus 605, 608

Cyclothone braueri 272

microdon 272

pallida 272

Cyema atrum 291

Cyemidae 291

Cylindrotermes 469, 493, 510

cyphergaster, Constrictermes 474

Cyprinogobius chrysosoma 52, 77

cystops, Rhinopoma hardwickei 595

dalmati, Strepsylla 541

danae, Chauliodus 277

Dangila, spilurus 63

darwini, Holochilus 647, 661, 663, 664

darwiniensis, Mastotermes 468

daubani, Aeletes 610

davisae, Strep sylla 546

davyi fulvus, Chilonycteris 697

Pteronotus 697

Dendrophilinae 601, 605, 616

Dendrophilus minutus 618 quattuordecimstriata 616

Dentispicotermes 494, 512

dentition, of Calamaria $173 \mathrm{ff}$ of sigmodont rodents $644 \mathrm{ff}$ of symmetrodont 690

dentritis, Bulimulus 319

derelictus ascendens, Bulimulus 317

Derichthyidae $\mathbf{2 9 0}$

Derichthys serpentina 290

Dermochelyidae 365

Dermogenys, ecological distribution 81 , 86 
pusillus 52, 54ff, 66 ecological distribution 82

Desmatodon 391

Desmochelyidae 365

Desmodus murinus 700

rotundus murinus 700

Diadecta 387

Diadectes 387, 391

Diadectidae 387,399

Diadectomorpha 386

distribution, geographic 390 geologic 390

Permian evolution 391

diaphana, Sternoptyx 277

Diaphus dofleini 286

fulgens 287

gemellari 287

metopoclampus 287

Diasparactus 391

Diatermes 507

diazi, Romerolagus 548

Didelphis albiventris 215, 243, 244 aurita 247

marsupialis 215

paraguayensis 243

difficilis amplus, Peromyscus 547

diluta, Epiphragmophora 327

Dimacrodon 393, 395, 398, 400

Dimetrodon 387, 393, 397 gigashomogenes 392,397

dinema, Photonectes 282

Dinocyon 44

Diogenichthys atlanticus 286

Dipnoi 388

Diretmidae 292

Diretmus argenteus 292

disjuncta, Margrethia 270

distribution, ecological, of fishes $81 \mathrm{ff}$ geographic, fossil reptiles $389 \mathrm{ff}$ vertical, of fishes $269 \mathrm{ff}$

Diversitermes 496, 514

döderleini, Calamaria 201

doerianense, Calamaria 199, 201

dofleini, Diaphus 286

Dolichorhinotermes 469, 493, 509

Dolichosoma 531, 532

Dolichosomatidae 531

doriae, Brachygobius 52, 77 food 77,85

Gobius 77

dorsalis, Vampyrops 407

Doryichthys brachyurus 55, 67

Drepanotermes 469, 473, 503, 510

Drymaeus canaliculatus 314

eucosmetus 331

interruptus 333

pamplonensis 314

poecilus 332,333

ponsonbyi 332

schmidti 314

serotinus 313,332

zilchi 333 dudleyi, Cryptotermes 492

Dunkard Formation 389

dunnei, Nesospiza wilkinsi 152

Duplidentitermes 511

dussumieri, Mugil 53, 68

ebriosus, Neotomys 646 vulturnus, Neotomys 646

Echiostoma tanneri 281

ectoparasitism, in beetles 211

Edaphosauria 387

distribution, geographic 390 geologic 390

Edaphosauria 387

Edaphosauridae 387, 399 evolution in Permian 392

Edaphosaurus 392

Edrabius 216, 218, 220, 254

alticolus 259

argentinus 258

pearsoni 219,258

peruanus 259

philippianus 212, 215, 254, 255

weiseri 255

Edriolychnus schmidti 299

egregia, Calamaria 201

Egretta alba 153

thula 152

Elaphe 169

elatus, Bulimulus 324

electa, Calamaria 201

Electrotermes 507

Electrotermitinae 507

elegans, Calamaria 190, 192, 201 Ptyssophorus 646

Eleotridae, ecological distribution 81-82

Eleotris fusca 52, 76 predator of 78

gymnopomus 76

melanosoma 52, 54, 76

food 76,85

porocephala 76

urophthalmus 77

Eleutheronema tetradactylum 55 food 85

elongatum, Gonostoma 270

elongatus, Barbus 62

emarginatus, Amblyopinus 239

Emberiza 150 tahapisi, horny palate 151

Emberizinae 149

Enchisthenes harti 700

Endodontidae 308

endospira, Bulimulus 336

Engraulis tri 56

enode, Platysoma 629

Enoploclytia walkeri schmidti 445

ensifer, Holocentrus 92

Eotermes 507

Epierus krujanensis 617 rubripes 618

Epinephalus nebulosus 55, 72 
food 85

Epiphragmophora atahualpa 327 claromphalos 327

clausomphalos 327

diluta 327

(Epiphragmophora) atahualpa 327

claromphalos 327

diluta 327

olssoni 336

patasensis 327

huanucensis 328

olssoni 336

patasensis 327

(Pilsbryna) clausomphalos 327 inferior 327

puntana 328

Eptesicus capensis 595

pusillus 595

tenuipinnis 595

equula, Leiognathus 74

equulus, Leiognathus 52ff, 74 food 74,85

Scomber 74

eremita, Nesocichla 145

Eremotermes 472, 480, 483, 510

Eretmochelys 366

imbricata 376

vertebrae 360

Erolia acuminata 154

erythrocephala, Ornithoctona 21

erythrogaster, Hirundo rustica 155

Ethiopian region, termite fauna of 484 , $508 \mathrm{ff}$

Euchilotermes 511

Euclastes melii $363,365,377$

eucosmetus, Drymaeus 331

Eucryptotermes 469, 492, 508

Euhamitermes 495, 510

Eurylister 628

Eurypharyngidae 289

Eurypharynx pelecanoides 289

Eurytermes 468, 493, 510

Euscaiotermes 513

Eustomias bibulbosus 282

bigelowi 282

obscurus 282

schmidti 282

Eutermellus 469, 487, 502, 515

Euxina moesta 433

Evenchelys macrurus 55, 58 food 85

everetti, Calamaria 192, 201

Nematabramis 53ff, 60 ecological distribution 82 food $60,84,88$

Evermannella atrata atlantica 284 balbo 284 indica melanoderma 285

Evermannellidae 284

evolution of cricetine dentition 643 of parasitic staphylinids 216 of rodents 217 of sigmodont dentition 643

extensus, Bulimulus 323

familiaris, Canis 42

Farancia 169

Faremusca 95

fasciola, Idiacanthus 283

Fastigitermes 512

fautini, Strepsylla 546

fauveli, Bacanius 605, 622, 624, 625

ferox, Stomias boa 278

Firmitermes 511

fishes, Bermuda deep sea $265 \mathrm{ff}$

complementary fresh-water 81

of North Borneo 47ff ecological distribution $81 \mathrm{ff}$ food of $84,87 \mathrm{ff}$

vertical distribution $269 \mathrm{ff}$

Flammeo, Holocentrus 92, 99

flavicans, Oryzomys 657

flaviventris, Serinus 139, 155

Flower Pot Formation 386 fauna of $393 \mathrm{ff}, 398$

fluviatilis, Tetraodon 54, 55, 80 Tetrodon 80

food, of Calamaria 174 of fishes $87 \mathrm{ff}$

Foramitermes 506, 515, 516

forestieri, Paromalus 619 Platylomalus 605, 608, 619

Forficulitermes 511

Formation, geologic, Abo 389

Admiral 389

Arroyo $389 \mathrm{ff}$

Belle Plains 389

Choza $389 \mathrm{ff}$

Clyde 389

Dunkard 389

Flower Pot 389

Hennessey 392

Moran 389

Putnam 389

San Angelo 389

Vale 389ff

formosana, Calamaria pavimentata 205

frantzii, Ortalis 20

Fringillaria tahapisi 150

Fringillinae 149

frontosum, Platysoma 631

Fruticicolidae 327, 336

fuegensis, Amblyopinus 216, 237

fulgens, Diaphus 287

fumosus, Vampyrops 405

fur, Termes 474

Furculitermes 511

fusca, Eleotris 52, 76 predator of 78

Poecilia 76

fuscipennis, Nothocercus 13

Gadidae 291

gahani, Amblyopinodes 248 
Galea musteloides 254

Galenomys garleppi 254

Gallinula 143

chloropus 143

garrula, Ortalis 20

Gastrocopta (Gastrocopta) wolfii 305

Gastromyzon borneensis 64

gastropicta, Calamaria margaritophora 204

gemellari, Diaphus 287

Gempylidae 298

Genuotermes 494, 512

Geoceras, Bulimulus 320

Geocichla 144

Geomyidae 218

Geopyrgus, Bulimulus 336

Geospiza 148

Gerres poeti 53, 74

food 74,85

gervaisi, Calamaria 169, 176, 178, 180, 201

Gibbotermes 488

gigashomogenes, Dimetrodon 392, 397

gimletti, Calamaria 196, 199, 201

girundica, Chelonia 366, 378

giurus, Glossogobius 52, 55, 78 food 78,85

Gobius 78

glacialis, Bathylagus 284

Glarichelys knorri 364, 377

Globitermes 469, 510

globulosa, Crax 16

Glossogobius giurus 52, 55, 78

food 78,85

predator of 68

Glossophaga soricina leachi 697

Glossotermes 479, 492, 509

Glyptotermes 468, 469, 473, 476-478, $484,492,498,507,508$

Gnathamitermes 469, 472, 473, 491, 510

Gobiidae, ecological distribution $81 \mathrm{ff}$

Gobiopterus brachypterus 55, 78

Gobius boddaerti 80

doriae 77

giurus 78

schlosseri 79

tentacularis 78

Gomphodus 459

Gonichthys cocco 286

Gonionenia, Nenia 308

Goniostomus subhybridus 314

Gonostoma bathyphilum 272 elongatum 270

Gonostomatidae 269

gordoni, Nesocichla 146

Gorgonopsia 387, 397

göringi, Calamaria 202

goudotii, Chamaepetes 21

Gough Island 142ff

goughensis, Nesospiza 148

Rowettia 142

horny palate 151 grabowskyi, Calamaria 178, 189, 202

gracilis, Calamaria 202

gracillima, Calamaria 196, 199, 202

Grallatotermes 477, 478, 481, 486, 487, 501, 503, 514

grandaeva, Chelone 345

Chelonia 345

Procolpochelys $345 \mathrm{ff}$

grandis robustior, Urotriainus 564

Syntermes 564

Urotriainus 564

Granopupa rhodia 429

gratus, Peromyscus truei 548

grayi, Calamaria 183, 185, 188, 207 vermiformis 188, 207

Green Sand 345

griswoldi, Calamaria 178, 183, 202 lumbricoidea 183, 202

guatemalensis, Peromyscus 544, 548

guernei, Photostomias 283

guianae, Holochilus 647, 659, 668

guttata, Ortalis 19

guttatus, Tinamus 12

gwinneri, Chelonia 364, 377

gymnocephalus, Ambassis 52, 54, 71

Lutjanus 71

gymnopomus, Butis 52, 55, 76 food 76,85

Eleotris 76

Habrocerinae 211

Habroconus (Pseudoguppya) semenlini 308

haedinus, Taphozous perforatus 580

Halacritus 611

halselli, Casea 396

hamatus, Bacanius 620

hamiltoni, Bulimulus 325

Thrissa 56

Thrissocles 53, 56, 58

food 56, 84

Hampala macrolepidota 52, 53, 62

ecological distribution 82

food 62,84

hancocki, Cotylorhynchus 396

Haptodus 392

hardwickei cystops, Rhinopoma 595

Harpage 95

rosea 95

harti, Artibeus 700

Enchisthenes 700

hasta, Lutjanus 73

Neoniphon 94

Pomadasys 53, 55, 73 food 73,85

hastatus, Holocentrus 92, 111

havilandi, Cryptotermes 487

Havilanditermes 481, 514

Helicinidae 304

Helicina (Helicina) chionea 305 peruviana 304

helleri, Vampyrops 411 
Hemicyon 44

hemigymnus, Argyropelecus 274

Hemirhamphus amblyurus 67

hemprichi, Otonycteris 595

Hennessey Formation 392

henseli, Amblyopinus 212, 247

Hershkovitz, Philip 9, 214

hershkovitzi, Amblyopinus 238

hershkovitzi, Tinamus osgoodi 10

Hesperomys 644

(Holochilus) brasiliensis 666 physodes 666 vulpinus 663

physodes 666

russatus 666

squamipes 666

vulpinus 657,664

Heteromys 218 anomalus 238

Heterotermes 468, 472, 473, 477-479, $483,484,490,493,499,509$

Heterotermitinae 516

hirtipes, Petalasoma 626

Hirtitermes 481, 514

Hirundo rustica erythrogaster 155

hispidus, Sigmodon 647

Hister, cyaneus 608

nanus 617

troglodytes 618

Histeridae $601 \mathrm{ff}$

Histerinae 605,626

Hodotermes 473, 484, 502, 509

Hodotermitidae $472,479,482,484,499$, 505-507, 509

Hodotermitinae 516

Hodotermopsis 470, 482, 509

hollandi, Calamaria 202

Holocentridae 91ff, $121 \mathrm{ff}$

auditory bulla 123

opisthotic bone 125

swim bladder 122

Holocentrinae 122

Holocentrum longipinne 100

perlatum 104, 107

osculum 104

productum 100

riparum 109

rostratum 99

Holocentrus 91ff, 121, 122

(Adioryx) 92, 109

bullisi 92

coruscus 92,100

hastatus 92

lacteoguttatus 92

microstomus 92

punctatissimus 92

suborbitalis 92,100

tiere 92

vexillarius 92,100

xantherythrus 92

ascensionis 93, 97, 100, 106, 122ff, 134

Atlantic species $91 \mathrm{ff}$ brachypterus 109

bullisi $91,97,113$

calcarifer 72

coruscus 97, 100, 112

(Flammeo) 92

laevis 92, 100

marianus 92

opercularis 92,100

sammara 92, 100

scythrops 92, 100

hastatus 111

leo 92

marianus 92, 96, 99

maxilla 92

meeki 95, 104, 109

osculus 91

punctatissimus 95

puncticulatus 112

rufus 91, 97, 102, 104, 122

sammara 94

sanctipauli 91, 100

(Sargocentron) 92

ensifer 92

leo 92

praslin 92

tieroides 92

spinosissimus 92

sicciferum 95, 109

spinifer 92

suborbitalis 92, 126, 132

tahiticum 95

tortugae 112

vexillarius $96,97,109$

Holocheilus 648

Holochilomy brasiliensis 662

Holochilus 639ff, 647, 648, 653

amazonicus 659,667

anguya 647

balnearum 656, 661, 665

berbicensis 661

brasiliensis $645,647,648,653,654$, $657,658,660,661,662,666$

amazonicus 667,669

balnearum 665

berbicensis 655,669 .

brasiliensis 662

guianae 668

incarum 668

leucogaster 648,665

nanus 666, 669

venezuelae 670

vulpinus 661,662

canellinus $647,661,666$

chacarius $655,656,663,664,665$

chrysogaster 661,662

darwini $647,661,663,664$

guianae $647,659,668$

incarum 659,668

leucogaster $647,661,666$

magnus $640,644,648,653,654,657$

multannus $647,661,663,664$

nanus $647,659,666,668$ 
physodes 647,666

russatus 647,661

sciureus $647,659,661,662,666,667$, 669

berbicensis 669

squamipes 647

venezuelae $661,669,670$

vulpinus $647,657,661,663$

Hololepta urvillei 630

Holotrachys 95, 122

lima 95

Homallotermes 469, 512

Hoplognathotermes 468, 485, 511

Hoplostethus cornutus 292

Hoplotermes 469, 493, 510

hosei, Calamaria 202

Hospitalitermes 477, 478, 481, 503, 514

huanucensis, Epiphragmophora 328

huilae, Amblyopinus 242

Hyalorhynchus natalensis 284

hygomi, Hygophum 286

Hygophum benoiti 285

hygomi 286

macrochir 286

reinhardti 285

hylocetes, Peromyscus 548

hyperythrus, Odontophorus 22

hypopius, Rhynchortyx cinctus 22

Hypotermes 513

hypsodonty 644

Hypsolenus 626

Hystricomorpha 217

Hystrichopsyllidae 541

ibis, Bubulcus 153

Ichthyocampus carce 54, 55, 67

Ichthyococcus ovatus 273

Idiacanthidae 283

Idiacanthus fasciola 283

Idister 626

mendax 626

morphon 626

ignobilis, Caranx 53, 55, 72

food 73,84

Scomber 72

Ilisha kampeni $52 \mathrm{ff}, \mathbf{5 7}$

food 57,84

imbricata, Eretmochelys 379

Incania, Nenia 307

incarum, Holochilus 659, 668 brasiliensis 668

Strophocheilus 331

incipiens, Proreithrodon 646

indica melanoderma, Evermannella 285

Indomalayan region, termite fauna of $479,508 \mathrm{ff}$

indragirica, Calamaria 202

inferior, Epiphragmophora clausomphalos 327

infundibulum, Bulimulus 320

infuscus, Vampyrops 405

ingolfianus, Nessorhamphus 291 inquilinus, Termes 474

insularum, Ampullarius 304

interrupta, Ambassis 52ff, 71

food 71,84

interruptus, Drymaeus 333

iostoma, Porphyrobaphe 331

iridescens, Calamaria gervaisi 182

Isospondyli, ecological distribution $81 \mathrm{ff}$ Ixodoidea 579

jacobus, Myripristis 93

jacqúaçu, Penelope 18

jaculator, Toxotes 74

jaculatrix, Sciaena 74

Toxotes 54, 74

food 74,85

jamaicensis, Artibeus 698

Jaminia chondriformis 419

ghilanensis 436

heptodon 423, 429ff

lamellifera $434 \mathrm{ff}$

libanica 429, 432ff, 435

ovularis $423,438 \mathrm{ff}$

saulcyi 416

septemdentata $421,422 \mathrm{ff}, 433$

sexdentata $436 \mathrm{ff}$

sulcidens $438 \mathrm{ff}$

tridens 440

jansoni, Myotyphlus 219, 259, 260

javanica, Calamaria 202

lineata, Calamaria 196, 202

jello, Sphyraena 54, 68

food 68,84

jelskii, Amblyopinus 212, 224

Thaumastus 309

jeudi, Calamaria 202

jocosensis, Bulimulus 322

joloensis, Calamaria 203

jouteli, Kalotermes 576

Jugositermes 511

julius, Nothocercus 12

kalinowskii, Amblyopinus 243

Nenia angrandi 306

Kalotermes 467, 468, 472, 473, 478, 483, $488,490,491,492,502,507,508$ jouteli 576

Kalotermitidae 476, 479, 484, 491, $505-508,516$

introductions 474

termitophiles 563, 575

Kalotermitinae 507

kampeni, Ilisha 52ff, 57 food 57, 84

Pellona 57

Karlschmidtia 327

lentiformis 328

klossi, Calamaria 203

knorri, Glarichelys 364, 377

koefoedi, Searsia 269

kofordi, Amplyopinus 231 
kretamensis, Tetraodon 52, 55, 81 food 81

krujanensis, Epierus 617

kuhli, Pipistrellus 595

Kutaflammeo 95

labecula, Peromyscus maniculatus 548

Labidosaurikos 391

Labidosaurus 391

Labiotermes 495, 514

Labritermes 510

Lacessititermes 481, 514

lacteoguttatus, Holocentrus 92

laetus, Saprinus 609

laeve, Platysoma 628

laevis, Aeletes 610

Holocentrus 92, 100

lamellifera, Jaminia 434ff

Lampadena bathyphila 288 chavesi 288

minima 288

Lampanyctus alatus 289

ater 288

cuprarius 288

photothorax 289

pusillus 289

supralateralis 289

taaningi 289

lankesteri, Neostethus 53, 69

lateralis, Calamaria 203

Lates calcarifer 53,72 food 84

Latisubulitermes 515

lautensis, Calamaria 178, 191, 203

leachi, Glossophaga soricina 697 Monophyllus 697

leathensis, Pteraspis 452

Leiognathus equula 74 equulus $52 \mathrm{ff}, 74$

food 74,85

lentiformis, Karlschmidtia 328

Lepidochelys $349,354,365$

carapace $374 \mathrm{ff}$

neurals 349

olivacea 376

plastron 377

scales, head 376

vertebrae 360

Lepidotermes 511

Lepospondyli 531

Leptocephalus pseudolatissimus 290 roule 290

splendens 290

Leptonycteris nivalis nivalis 698

Leuciscus sumatranus 60

leucocephala, Calamaria 169, 176, 178, 193, 203

leucogaster, Calamaria 169, 176, 179, 203 dentition $172 \mathrm{ff}$

Holochilus 647, 661, 666

Holochilus brasiliensis 648,665

Mus (Holochilus) 648, 660, 666
Leucopitermes 477, 478, 489, 502, 514

leucopogon, Astronesthes 278

leucops, Subulitermes 502

leucopus, Rhamphoberyx 94

levipes, Peromyscus boylii 545

libanica, Jaminia $432 \mathrm{ff}$

lilium parvidens, Sturnira 700

lilliputianus, Acritus 612

lima, Holotrachys 95

limbs, of Cheloniidae 364, 378

Limnopomus, Ampullarius 303

linearis, Phlegethontia 531

lineata, Calamaria javanica 196, 202

lineatus, Vampyrops 408, 409

linnaei, Calamaria 171, 195, 203

Linophryne arborifera 300

bicornis 300

Lissoacme, Bulimulus $317 \mathrm{ff}$

lobatus, Rhinolophus 595

London Clay 345

longibarbatus, Macrostomias 279

longicauda, Bartramia 154

longicaudatus, Oryzomys 648

longiceps, Chelone 345

longidens, Caulolepis 292

Longipeditermes 469, 481, 514

longipes, Aeletes 614

longipinne, Holocentrum 100

longitudinalis, Bulimulus 316

longus, Amblyopinus 234

Lophodolus acanthognathus 299

Lophogobius chrysosoma 77

lowi, Calamaria 178, 196, 199, 203

Loxops 148

lumbricoidea, Calamaria 178, 183, 189, 203

griswoldi, Calamaria 183, 202

lumholtzi, Calamaria 203

lutescens, Mus 648

Lutianus argentimaculatus 54, 55, 73 food 84,85

Lutjanus gymnocephalus 71 hasta 73

Lysorophidae 531

Macraster elegans 447

macrochir, Hygophum 286

macrolepidota, Hampala 52, 53, 62

ecological distribution 82

food 62,84

macrolychnus, Neonesthes 278

macropus, Ophthalmopelton 284

Macrosternus montrouzieri 627

Macrostomias calosoma 279

longibarbatus 279

Macrosubulitermes 503, 515

Macrotermes 473, 484, 486, 507, 513

Macrotermitinae 486, 505, 506, 513, 516

macrurus, Evenchelys 55, 58

food 85

Muraena 58

macularia, Actitis 154 
maculatus, Anthias 73

Pomadasys 53, 73

food 73,85

magnus, Holochilus 640, 644, 648, 653, 654,657

major, Chaerephon 595

Opheodrys 174

Tinamus 9

Malacosteidae 283

Malagasitermes 469, 489, 501, 514

Malagasy region, termite fauna of 487ff, $502,508 \mathrm{ff}$

Manchurodon 692

maniculatus labecula, Peromyscus 548

margaritophora, Calamaria 191, 192, 203 gastropicta 204

marginalis, Rhinotermes 493

Margrethia disjuncta 270

obtusirostra 270

marianus, Holocentrus 92, 96

marmoratus, Odontophorus gujanensis 21 marmosae, Amblyopinus 247

martinica, Porphyrula 153

Mastotermes 476, 490, 498, 507, 508 darwiniensis 468

Mastotermitidae 479, 490, 491, 505-508, 516

maxilla, Holocentrus 92

maximus vestitus, Strophocheilus 309

mazonensis, Phlegethontia 523

mearnsi, Calamaria 204

mecheli, Calamaria 204

meeki, Holocentrus 95, 104, 109

Megaderma cor 580, 595

Megagnathotermes 468, 485, 511

Megalobulimus, Strophocheilus 309

megalophylla, Mormoops 697

megalotis mexicana, Micronycteris 697

Megamblyopinus $212,215,216,218$, 222,251

germaini 254

mniszechi 216, 219, 251

mehelyi, Rhinolophus 595

Melamphaes anthrax 297

microps 296

mizolepis 297

opisthopterus 296

robustus 297

Melamphaidae 296

Melanocetidae 299

Melanocetus murrayi 299

Melanodera 144, 147-149

melanodera 149

horny palate 151

melanoderma, Evermannella indica 285

Melanonus unipennis 291

melanosoma, Eleotris 52, 54, 76 food 76,85

Ophicephalus 54, 71

ecological distribution 82

food 71,84

Melanostomiatidae 281
Melanosuchus niger 339

melanota, Calamaria 204

melasoma, Ophicephalus 71

melii, Euclastes $363,365,377$

Melursus ursinus 25

mendax, Idister 626

Metachirus opossum 247

metallicus, Bathophilus 281

Merostichis 656

metopoclampus, Diaphus 287

mexicana, Chilonycteris rubiginosa 696

Micronycteris megalotis 697

Mexico, bats from $695 \mathrm{ff}$

Microborus, Strophocheilus 330

microcephalus, Neonesthes 278

Microcerotermes 467, 469, 472, 473, 478, $480,483,485,488,493,507,510$

microdon, Cyclothone 272

Microhodotermes 471, 473, 477, 482, 484, 502, 509

Micronycteris megalotis mexicana 697

microphthalma, Syntermorpha 572

microphyllum, Rhinopoma 595

microps, Melamphaes 296

Microsauria 531

microstomus, Holocentrus 92

Microtermes 473, 486, 489, 501, 513

Milleretidae 392

milloti, Eutermes 501

Mimeutermes 487, 489, 502, 514

mina, Strepsylla 546

mindorensis, Calamaria 204

minima, Lampadena 288

Miniopterus natalensis arenarius $\mathbf{5 9 5}$

minuta, Carcinops 618

minutus, Dendrophilus 618

Miocene Marl 345

Miotermes 507

mira, Ortalis 20

Mirocapritermes 512

mizolepis, Melamphaes 297

mjobergi, Calamaria 204

mniszechi, Megamblyopinus 216, 219, 251

modestus, Bulimulus 318

Zonibyx 154

molesti, Urotriainus 564

molestus, Syntermes 562, 564, 571

Molgophidae 531

Molossus rufus nigricans 701

rufus rufus 701

sinaloae 701

Monopterus alba 53, 59

montagnii, Penelope 18

monticolus, Amblyopinus 228

montivagans, Amblyopinus 240

montrouzieri, Apobletes 605, 607, 627

Macrosternus 627

Platysoma 605, 607, 629

Moran Formation 389

Mormoops megalophylla megalophylla 697 
morphon, Idister 626

Mucrotermes 511

muelleri, Calamaria 192, 204

Mugil caeruleomaculatus 68

dussumieri 53, 68

Mullerister 620

multannus, Holochilus 647, 661, 663 664

Muraena alba 59 macrurus 58

murinus, Desmodus 700

Desmodus rotundus 700

Muroidea 218

murrayi, Melanocetus 299

Mus agilis 648

angouya 660,662

brasiliensis $660,662-664$

(Holochilus) anguya 648, 660, 666

(Holochilus) leucogaster 648, 660

lutescens 648

physodes 660,665

simpsoni 648

vulpinus 660,662

mutabilis, Bulimulus 334

Myctophidae 285

mydas, Chelonia 360

Myomorpha 217

Myotyphlus 211, 215, 217, 218, 259 jansoni 219, 259, 260

Myripristinae 122

auditory bulla 124

opisthotic bone 125

swim bladder 123

Myripristis 96

argyromus 136

jacobus 93,121

occidentalis 93, 121

Mystus nemurus 53ff, 65

ecological distribution 82,86

food 66,84

nalua, Ambassis 53, 72

Chanda 72

nanus, Hister 617

Holochilus 647, 659, 666, 668 brasiliensis 666

Nasutitermes 468, 469, 472, 473, 477, $478,480,481,483,486,489,495$, 496,514

Nasutitermitinae 486, 487, 495, 505, $514,516,562$

nasutus, Nesiarchus 298

natalensis, arenarius, Miniopterus 595

Hyalorhynchus 284

Rhynchohyalus 284

Natrix 169

nuchalis 174

Nearctic region, termite fauna of 489 , 508 ff

nebulosus, Epinephelus 55, 72 food 85
Serranus 72

Nectomys 654, 670

squamipes 249,653

Nectridia 531

Nematabramis everetti $53 \mathrm{ff}, 60$

ecological distribution 82

food $60,84,88$

predator of 62

Nemichthyidae 291

Nemichthys scolopaceus 291

nemorensis, Bulimulus 316

nemurus, Bagrus 65

Mystus 53ff, 65

ecological distribution 82,86

food 66,84

Nenia angrandi kalinowskii 306 urubambensis 306

(Gonionenia) ueyrauchi 308

(Incania) warszewiczi 307

(Neniatracta) adusta 305

tumens 305

Neniatracta, Nenia 305

Neobacanius 620

Neocapritermes $469,495,512$

Neomyripristis 94 amaenus 94

Neonesthes macrolychnus 278 microcephalus 278

Neoniphon 94 armatus 94

hasta 94

Neopetraeus cora unicolor 335

cretaceus 335

platycheilus 311

rhodolarynx 311

Neopsyllinae 541

Neostethus bicornis 68

borneensis 52, 54ff, 69

lankesteri 53, 69

Neotermes $467,468,470,472,478,483$, $490,492,498,507,508,576$

Neotomodon 644 alstoni 547

Neotomys $639,640,644,645,646$ ebriosus ebriosus 646 vulturnus 646

Neotropical region, termite fauna of $491,508 \mathrm{ff}$

Nesiarchus nasutus 298

nesiotis, Porphyriornis 143

Nesocichla 144ff

eremita eremita 145

gordoni 146

procax 146

Nesospiza 157, 158

acunhae 147

acunhae 152

horny palate 151

questi 152

goughensis 148

wilkinsi 147

dunnei 152 
wilkinsi 150

Nessorhamphidae 291

Nessorhamphus ingolfianus 291

New Caledonia, Histeridae of $601 \mathrm{ff}$ nicholsi, Casea 396

niger, Melanosuchus 339

pluriradiatus, Chiasmodon 297

nigricans, Molossus rufus 701

nigro-ocellatum, Centrobranchus 286

nimbraria, Vinciguerria 273

Nitiditermes 511

nitiduloides, Saprinus 610

nivalis, Ischnoglossa 698

Leptonycteris 698

Noditermes 511

norvegicus, Rattus 648

Nothocercus bonapartei bonapartei 12 fuscipennis 13

julius 12

venezuelensis 14

Notolychnus valdiviae 288

novaescotiae, Cephalaspis 454

novae-scotiae, Pteraspis 452

nubilus, Ampullarius 303

nuchalis, Calamaria 204

Natrix 174

nudiventris, Taphozous 595

Nycteris thebaica 595

obeliscus, Bulimulus 322

obscura, Penelope 18

obscurus, Eustomias 282

obtusirostra, Margrethia 270

Obtusitermes 497, 514

Occasitermes 503, 514

occidentalis, Myripristis 93

occipitalis, Calamaria 204

Occulitermes 503, 515

Odontophorus erythrops parambae 21 gujanensis buckleyi 21 marmoratus 21

hyperythrus 22

Odontotermes 469, 472, 473, 513

olivacea, Lepidochelys 376

olivascens, Sicalis 149

horny palate 151

olivensis, Reithrodon 646

olssoni, Epiphragmophora 336

onca, Panthera 42

Onchus 458, 459

murchisoni 449

Oneirodidae 299

opercularis, Holocentrus 92, 100

Opheodrys major 174

Ophiacodonta 387

distribution, geographic 390 geologic 390

Ophicephalus melanosoma 54,71 ecological distribution 82 food 71,84 melasoma 71

Ophiderpeton 531, 532
Ophiderpetonidae 531

Ophiocara porocephala 52, 76 Ophiocephalus melanosoma 71

Ophiotermes 494, 511

Ophthalmopelton macropus 284

opisthopterus, Melamphaes 296

Oriensubulitermes 515

ornata, Calamaria 204

ornatus, Tremarctos $25 \mathrm{ff}$

jaw mechanics 40

mandibular articulation 38

muscles 32

salivary glands 31

skull 26

tongue and oral vestibule 29

vessels and nerves 31

Ornithoctona erythrocephala 21

Ortalis garrula chocoensis 20 garrula 20 mira 20

guttata caucae 19

columbiana 19 guttata 19

Orthognathotermes 469, 494, 512

Orthotermes 485, 512

Oryzomys 644

albigularis 214, 242

angouya 654

buccinatus 654,660

flavicans 657

keaysi 239

longicaudatus 654

sp. 251

oryzomysi, Amblyopinus 240

osculus, Holocentrus 91, 104

osgoodi, Tinamus 10

Ostariophysi 127

ecological distribution $82 \mathrm{ff}$

Osteochilus spilurus 53, 63 ecological distribution 82

Ostichthyes 96

Ostichthys 122 trachypoma 123

Otonycteris hemprichi hemprichi 595

ovatus, Ichthyococcus 273

ovularis, Jaminia $438 \mathrm{ff}$

Oxyeleotris urophthalmus 52, 77 food 77,85

Oxymycteris judex 249 rufus 249

Oxyurichthys tentacularis 55, 78

pacae, Amblyopinus 244

Pachyotus 595

pacificum, Platysoma 605, 607, 629

pacificus, Platylister 629

Palaearctic region, termite fauna of 481 , $508 \mathrm{ff}$

Palaeastacus walkeri 445

palans, Carcinops 618

pallida, Cyclothone 272

pamplonensis, Drymaeus 314 
Panthera onca, weights of jaw muscles 42 Pantotheria 689

Papio maimon 44

Papuan region, termite fauna of $477 \mathrm{ff}$, $503,508 \mathrm{ff}$

papyraceus, Ampullarius 303

Paracapritermes 469, 477, 503, 512

Paracornitermes 468, 473, 487, 495, 502, 514

parallelism $385 \mathrm{ff}$

Paramastus 429

parambae, Odontophorus erythrops 21

Paramyidae 217

Paraneotermes 473, 490, 508

Parapocryptes rictuosus 55, 80 food 80,85

Parareptilia 387

Parastylotermes 507

Parasubulitermes 515

Pareiasauridae 387, 392, 397ff

parnelli, Chilonycteris 696

Paromalus forestieri 619 pumilio 616 quattuordecimstriata 617 troglodytes 618

Parophiocephalus unimaculata 70

Parotermes 490, 507

Parrhinotermes 478, 500, 509

parvidens, Sturnira lilium 700

Parvitermes 497, 514

patagonicus, Phrygilus 149 horny palate 151

patasensis, Epiphragmophora 327

pavimentata, Calamaria 193, 204 formosana 205 pavimentata 204

Pearson, Oliver P. 215, 252, 254

pearsoni, Edrabius 219, 258

pedialota, Bonapartia 269

pelamidis, Rhynchichthys 93

pelecanoides, Eurypharynx 289

Pellona kampeni 57

Pelycosauria 387,400

distribution, geographic 390 geologic 390

pendleburyi, Calamaria 178, 191, 192, 205

Pene 429

Penelope montagnii montagnii 18 purpurascens aequatorialis 17 jacquiaçu 18

Pennsylvanian, Late 387 fauna 390

Perca ascensionis 100 rufa 104, 107

Percesoces, ecological distribution 81-83

Percoidea, ecological distribution 81-83 perforatus, Bulimulus 320 Taphozous 580, 595

Pericapritermes 469, 486, 495, 501, 511

Periophthalmus schlosseri 79 schlosseri 55, 79 variabilis 55,79

perlatum, Holocentrum 104, 107

Permian fauna $385 \mathrm{ff}$

Peromyscus 644

boylii levipes 545

difficilis amplus 547

guatemalensis 231, 544, 548

hylocetes 548

maniculatus labecula 548

truei gratus 548

Peronaeus, Bulimulus 321

perroudi, Platysoma 629

personata, Chilonycteris 696

perspicax, Penelope 17

Peru, shells from $304 \mathrm{ff}$

peruanus, Edrabius 259

Peruvian Zoological Expedition (1953) $221,304 \mathrm{ff}$

(1954) $330 \mathrm{ff}$

peruviana, Helicina 304

Succinea 305

peruvianus, Amblyopinodes 250

peruvianus, Radiodiscus 308

Petalasoma hirtipes 626

pfefferi, Calamaria 205

phaeocheilus, Bulimulus 334

philippianus, Edrabius 212, 215, 254, 255

Phlegethontia 523ff

linearis 531

mazonensis $523 \mathrm{ff}$

serpens 531

Phlegethontidae 524, 531

Photonectes dinema 282

Photostomias guernei 283

photothorax, Lampanyctus 289

Phrygilus patagonicus 149 horny palate 151

Phyllostoma lineatum 403, 409

Phyllotis 644

boliviensis 254

darwini 234

sublimus 234

phylogeny of Cheloniidae $363 \mathrm{ff}$

physodes, Hesperomys 666

Holochilus 647, 666

Mus 660, 665

Reithrodon 646

piceus, Amblyopinodes 248

Pilotermes 511

Pilsbryna, Epiphragmophora 327

Pimelodus sagor 64

Pipile cumanensis cumanensis 21

Pipistrellus kuhli kuhli 595

Plaesius 626

Planicapritermes 495, 512

plastron, of Cheloniidae 377 of Procolpochelys 357

platycheilus, Neopetraeus 311

Platylister pacificus 629 urvillei 630

Platylomalus forestieri 605, 608, 619 
saucius 619

umbilicatus 619

victoriae 619

Platypsyllus castoris 248

Platysoma 627

abruptum 629

connexum 605, 607, 628, 629

contiguum 629

enode 629

frontosum 631

laere 628

montrouzieri $605,607,629$

pacificum $605,607,629$

perroudi 629

(Platylister) montrouzieri 629 urvillei 630

(Platysoma) connexum 628

montrouzieri 629

pacificum 629

urvillei 630

querulum 628

soronense 631

urvillei $605,608,630$

ranum 631

plebius, Polydactylus 55, 70

food 85

Plecotus auritus christiei 595

Plectognathi, ecological distribution 83

Plectrypops 96, 122

Plesiochelyidae 369

plicata, Balantiopteryx 695

pliopterus, Chirostomias 281

Pliotermes 507

pluriradiatus, Chiasmodon niger 297

Poecilia fusca 76

poecilopus, Rhamphoberyx 93

poecilus, Drymaeus 332

poeti, Gerres 53, 74

food 74,85

polillensis, Calamaria 205

Poliospiza 147

burtoni 149

horny palate 151

politus, Aeletes 610

Polydactylus plebius 55, 70

food 85

Polynemus tetradactylum 69

Poly poraceae 561

Pomadasys hasta 53, 55, 73

food 73,85

maculatus 53, 73

food 74,85

Pomiatus olivieri 433

ponsonbyi, Drymaeus 332

popelairianus, Strophocheilus 309

porocephala, Eleotris 76 Ophiocara 52, 76

Porotermes 468, 470, 471, 476, 484, 491, $492,498,502,509$

Porotermitinae 516

porphyreus, Thaumastus 309

Porphyrio 143
Porphyriornis nesiotis comeri 142, 143 nesiotis 143

Porphyrobaphe iostoma 331

Porphyrula 143

martinica 153

Postsubulitermes 515

Potamopyrgus subgradatus 304

poweriae, Vinciguerria 273

prakkei, Calamaria 190, 192, 205

praslin, Holocentrus 92

Proboscitermes 512

Procapritermes 511

procax, Nesocichla 146

Procolophonidae 387, 392, 400

Procolpochelys grandaeva $345 \mathrm{ff}$

carapace 349

phylogeny $363 \mathrm{ff}$

plastron 357

vertebrae 360

Procornitermes 472, 473, 495, 514

Procryptotermes 472, 473, 478, 490, 492, 498, 508

Procubitermes 511

productum, Holocentrum 100

productus, Termitopteryx 572, 575

Proelectrotermes 507

Profastigitermes 511

Prohamitermes 493, 510

Prokalotermes 507

Promirotermes 469, 485, 486, 494, 512

Proreithrodon 646

chapalmalense 646

incipiens 646

Prorhinotermes 471, 477, 478, 479, 488, $489,493,500,509$ canalifrons 488

proteiformis, Bulimulus 335

Protermes 513

Proterothyris 391

proteus, Bulimulus 335

Protoca pritermes 503, 511

Protoglypius, Bulimulus 336

Protohamitermes 468, 480, 510

Protostegidae 365

proximus, Saprinus 610

Psammosteus cf. anglicus 449

Psammotermes 473, 479, 483, 484, 499, 509

roeltzkowi 488

Psammotermitinae 516

Pseudacanthotermes 486, 513

Pseudocapritermes 512

Pseudogobius bikolanus 52, 54, 79

sp. A 55, 79

sp. B 52,79

Pseudoguppya, Habroconus 308

pseudolatissimus, Leptocephalus 290

Pseudomicrotermes 510

psilotis, Chilonycteris 696

Psittirostra 148

Psophocichla 144

Pteraspis cf. crouchi 449 
leathensis 452

novae-scotiae 452

vogti 452

whitei 450

Pteronotus davyi fulvus 697

Pteryx 562

Ptiliidae 561ff

habitat 561

termitophilous adaptations 563

Ptinella 562

Ptyssophorus 646

elegans 646

rotundatus 646

Pugnititermes 511

pumilio, Carcinops 605, 608, 616, 619

Carcinus 616

Paromalus 616

punae, Amblyopinus 227

punctatissimus, Holocentrus 92, 95

puncticulatus, Holocentrus 112

punctiger, Abraeus 624

Bacanius 605, 606, 622, 624, 625

puntana, Epiphragmophora 328

Puntius sealei 52ff, 62

ecological distribution 82

food 62,84

predator of 62

Pupa chondriformis 419

michoni 432

septemdentata 422

pupiformis, Bulimulus 325

Puppigerus 345

purpurascens, Penelope 17

pusillus, Dermogenys 52, 54ff, 66

Eptesicus 595

Lampanyctus 289

Putnam Formation 389

Pycnopteryx 562, 563, 567 schmidti $562,567,570$ sp., 572

pyrgidium, Bulimulus 321

Quasitermes 469, 488, 512

quattuordecimstriata, Carcinops 616

Dendrophilus 616

Paromalus 617

Quechua, Thaumastus 331

Quediini 211

querulum, Platysoma 628

questi, Nesospiza acunhae 152

quinquetaeniata, Calamaria 205

radiation, adaptive 388,399

Radiconus, Radiodiscus 308

Radiodiscus (Radioconus) andium 308

(Radiodiscus) peruvianus 308

Rasbora sumatrana $52 \mathrm{ff}, 60$ ecological distribution 82

food $60,84,88$

ratada $655 \mathrm{ff}$

rats, marsh $639 \mathrm{ff}$

Rattus norvegicus 648 raveni, Calamaria 205

rebentischi, Calamaria 205

recifinus, Vampyrops 413

reconditus, Bulimulus 320

reinhardti, Hygophum 285

Reithrodon 639, 640, 644, 646

auritus 646

caurinus 646

cuniculoides 646

olivensis 646

physodes 646

typicus 646

remingtoni, Bacanius $605,606,622$, 624

reptiles of Permian 385ff

geographic distribution 389

Reticulitermes 470, 471, 483, 489, 490, 507,509

revinctus, Bulimulus 316

Rhamphoberyx 93

leucopus 94

poecilopus 93

Rhinoberyx 93

chryseus 93

Rhinolophus clivosus brachygnathus 595 lobatus 595 mehelyi 595

Rhinopoma hardwickei cystops 595 microphyllum 595

Rhinotermes 469, 493, 509 marginalis 493

Rhinotermitidae 476, 479, 505-507, 509 introductions 474

Rhinotermitinae 516

rhodolarynx, Neopetraeus 311

Rhynchichthys 93

brachyrhynchus 93

pelamidis 93

Rhynchohyalus natalensis 284

Rhynchortyx cinctus hypopius 22

Rhynchotermes 468, 495, 514

rictuosus, A pocryptes 80

Parapocryptes 55, 80 food 80,85

ridley turtles 345

riparum, Holocentrum 109

rivasii, Bulimulus 336

robustior, Urotriainus grandis 564

robustus, Melamphaes 297

rodents, cricetine 639 dentition $643 \mathrm{ff}$

sigmodont $639 \mathrm{ff}, 646$

dentition 643

skull 640

rogersi, Atlantisia 142

romeri, Cotylorhynchus 396

Romeria 391

Romerolagus diazi 548

rosea, Harpage 95

rostratum, Holocentrum 99

Rostrotermes 511

Rothia 393, 398 
Rotunditermes 496, 514

rotundus murinus, Desmodus 700

roulei, Leptocephalus 290

Rousettus aegyptiacus aegyptiacus 595

Rowettia 144, 147-150, 158

goughensis 142

horny palate 151

rubiginosa, Chilonycteris 696 mexicana, Chilonycteris 696

rubra, Crax 17

Sciaena 104

rubripes, Carcinops 618, 619

Epierus 618

rudistriatus, Bulimulus 318

rufa, Perca 104, 107

ruficeps, Tinamus major 10

rufus, Holocentrus 91, 102, 104, $122 \mathrm{ff}$ Molossus 701

Rugitermes 477, 478, 491, 492, 498, 508 arthuri-muelleri 576

ruppelli, Tadarida teniotis 595

russatus, Hesperomys 666

Holochilus 647,661

sagor, Pimelodus 64

Tachysurus 55, 64

food 85

sammara, Holocentrus 92, 94, 100

San Angelo Formation 385

fauna of $393 \mathrm{ff}, 398$

sanborni, Amblyopinus 238

sanctipauli, Holocentrus 91, 100

Saprininae 605, 608

Saprinus artensis 605, 608, 609

australasiae 609

cyaneocupreus 610

cyaneus 605, 608

laetus 609

nitiduloides 610

proximus 610

speciosus 609

subnitidus 605, 608, 610

tasmanicus 609

Sargocentron, Holocentrus 92

saturatus, Tinamus major 9

saucius, Platylomalus 619

saulcyi, Bulimus 416

Jaminia 416

scales, head, in Cheloniidae 376

Scatophagus argus 52, 54, 55, 75

Schedorhinotermes 469, 478, 479, 484, $493,500,509$

schlegeli, Calamaria 205

schlosseri, Gobius 79

Periophthalmus 79 schlosseri 55,79

schmidti, Acritus 605, 607, 612, 614

Calamaria 174, 178, 197, 205

Drymaeus 314.

Enoploclytia walkeri 445

Pycnopteryx 562, 567, 570

Strepsylla 544
Thaumastus 309

schmidti, Edriolychnus 299

Eustomias 282

scholvienia, Thaumastus 309

Sciaena argentimaculata 73

jaculatrix 74

rubra 104

sciureus berbicensis, Holochilus 669

Holochilus 647, 659, 661, 666, 667, 669

Sciuromorpha 217

sclerops apaporiensis, Caiman 340

Caiman 339

scolodonta, Streptaxis 329

scolopaceus, Nemichthys 291

Scomber equulus 74

ignobilis 72

Scopelarchidae 284

Scopelarchus anale 284

Scutalus, Bulimulus 315ff, 334

scutulatus, Bulimulus 319

scythrops, Holocentrus 92, 100

sealei, Barbodes 62

Puntius 52ff, 62

ecological distribution 82

food 62,84

Searsia koefoedi 269

Searsidae 269

semenlini, Habroconus 308

semiannulata, Calamaria 205

septemdentata, Jaminia 421, $422 \mathrm{ff}$

septentrionalis, Calamaria 169, 178, 194, 206

Serinus 147

flaviventris 139,155

sulphuratus 149

horny palate 151

serotinus, Drymaeus 313, 332

serpens,'Phlegethontia 531

serpentina, Derichthys 290

Serranus nebulosus 72

Serritermes 515

Serritermitinae 497, 505, 506, 515, 516

Serrivomer beani 291

brevidentatus 291

Serrivomeridae 291

sexdentata, Jaminia $436 \mathrm{ff}$

sharks, xenacanth 388

Sicalis olivascens 149

horny palate 151

sicciferum, Holocentrus 95, 109

Sigmodon 639, 640, 644, 645, 647, 648, 652,653

hispidus 647

vulpinus 657

Sigmomys 647

Silvestritermes 515

simalurensis, Calamaria 189, 190, 192, 206

similis, Amblyopinodes 251

simonyi, Benthodesmus 298

simpsoni, Mus 648

sinaloae, Molossus 700 
Siphonaptera 541

sismondai, Chelonia 366, 378

sloanei, Chauliodus 277

smithi, Calamaria 206

snyderi, Syntermes 562, 570, 572

soborna, Corica $52,54,57$

Solenichthyes, ecological distribution 83 sondaica, Calamaria 206

soricina leachi, Glossophaga 697

soronense, Platysoma 631

soui, Crypturellus 15

Spalacotheriidae 689

Spalacotherium 690, 692

Spalacotheroides 690,692

bridwelli 690

dentition $690 \mathrm{ff}$

specialization, in Calamaria 168

speciosus, Saprinus 609

Speculitermes 480, 493, 500, 510, 567

termitophiles of 562

Sphaerotermes 513

Sphenacodon 392

Sphenacodontia 387

distribution, geographic 390 geologic 390

Sphyraena jello 54, 68 food 68,84

Spicotermes 494, 511

spilurus, Dangila 63

Osteochilus 53, 63 ecological distribution 82

spinalis, Achalinus 174

spinifer, Holocentrus 92

spinipennis, Amblyopinus 234

Spinitermes 485, 494, 511

spinosissimus, Holocentrus 92

Spinus 147

spinus 149

horny palate 151

Spirosulcatus, Bulimulus 336

splendens, Leptocephalus 290

squamipes, Hesperomys 666

Holochilus 647

Nectomys 653

stahlknechti, Calamaria 192, 206

Staphylininae 211, 221

Stegocephali 531

Stephanian fauna $389 \mathrm{ff}$

Steppesaurus $393,397 \mathrm{ff}$

Sternaulax 605, 626

caledoniae $605,607,626$

zealandica 626

Sternoptychidae 274

vertical distribution 275

Sternoptyx diaphana 277

Stolephorus commersoni 52,56 food 56,84

tri 54, 56 food 84

Stolotermes 468, 470, 471, 476, 484, 498, 502,509
Stolotermitinae 516

Stomias boa ferox 278

brevibarbatus 279

Stomiatidae 278

Strepsylla $541 \mathrm{ff}$

dalmati 541

davisae 546

fautini 546

mina 541

schmidti 544

taluna 546

villai 546

Streptaxidae 329

Streptaxis (Scolodonta) andivaga 329

strongylura, Belone 66

strongylurus, Tylosurus 53, 66 food 84

Strophocheilidae 309,330

Strophocheilus incarum 331 maximus vestitus 309

(Microborus) tenuis 330 popelairianus 309

tenuis 330

Sturnira lilium parvidens 700

styani, Trirhinopholis 174

Stylotermes 509

Stylotermitinae 516

subgradatus, Potamopyrgus 304

subhybridus, Goniostomus 314

subjussieui, Bulimulus 336

subnitidus, Saprinus 605, 608, 610

suborbitalis, Holocentrus 92, 100, 126, 132

subulidens, Caulolepis 293

Subulitermes $469,481,487,489,496$, $497,502,515$

leucops 502

undecimus 502

Succinea aequinoctialis 305

peruviana 305

Succineidae 305

sulcidens, Jaminia $438 \mathrm{ff}$

sulcisternus, Bacanius 620

sulphuratus, Serinus 149

horny palate 151

suluensis, Calamaria 178, 190, 192, 206

sumatrana, Calamaria 191, 192, 206

Rasbora 52ff, 60 ecological distribution 82

food $60,84,88$

sumatranus, Leuciscus 60

supralateralis, Lampanyctus 289

Symmetrodonta 689

Sympholis 168

Synacanthotermes 468, 473, 513

Synapsida 387

Synbranchoidea, ecological distribution 83

Synentognathi, ecological distribution 83

Syngnathus brachyurus 67 carce 67 
Synhamitermes 510

Syntermes 468, 473, 495, 514 grandis 564

molestus 562, 564, 571

snyderi 562,570

termitophiles of 561

Syntermorpha microphthalma 572

Systrophia (Systrophiella) andivaga 308

Systrophiella, Systrophia 308

Systrophiidae 308

taaningi, Lampanyctus 289

Tachysurus caelatus 55, 64 food 85

sagor 55,64

food 85

venosus 55,65

food 84

Tadarida aegyptiaca aegyptiaca 595 teniotis ruppelli 595

tahapisi, Emberiza, horny palate 151

tahiticum, Holocentrus 95

taluna, Strepsylla 546, 547

tanneri, Echiostoma 281

Taphozous nudiventris 595

perforatus 580

haedinus 580, 595

perforatus 595

Tapinocephalia 387, 393, 397

Tappenosaurus 395, 397

Tarditermes 515

tasmanicus, Saprinus 609

teniotis ruppelli, Tadarida 595

tentacularis, Gobius 78

Oxyurichthys 55, 78

tenuipinnis, Eptesicus 595

Tenuirostritermes 472, 481, 491, 496, 497,514

tenuis, Strophocheilus 330

Termes 468, 469, 473, 477, 478, 480, $485,488,494,507,512$

fur 474

inquilinus 474

termites, zoogeography $465 \mathrm{ff}$

Termitidae 479, 505-507, 510-512

Termitinae $485,487,494,505,506,511$, 512,516

Termitogeton 509

Termitogetoninae 516

termitophiles, of Kalotermitidae 563 of Speculitermes 562 of Syntermes 561, 562

Termitopteryx 563, 572 productus $572, \mathbf{5 7 5}$ sp. 576

Termopsinae 482, 516

Termopsis 482,507

tetradactylum, Eleutheronema 55, 69 food 85

Polynemus 69

Tetragonuridae 298

Tetragonurus atlanticus 298
Tetraodon, ecological distribution 81,86

fluviatilis 54, 55, 80

ecological distribution 82

food 80,85

kretamensis $52,55,81$

ecological distribution 82

food 81,85

Tetrodon fluviatilis 80

teysmanni, Clarias 52, 54, 65 ecological distribution 82

food 65,84

Thaptomys nigrita 249

Thaumastus bitaeniatus 309

conspicuus 331

jelskii 309

porphyreus 309

(Quechua) conspicuus 331

schmidti 309

thebaica, Nycteris 595

Thecophora 368

Therapsida $387,392,400$

distribution, geographic 390 geologic 390

origin 388

Theria 689

Theriodonta 387, 397

distribution, geographic 390 geologic 390

origin 388

Therocephalia 387,397

Thomasomys 232, 644 aureus nicefori 232 laniger 239

Thoracotermes 511

Thrissa hamiltoni 56

Thrissocles hamiltoni $53,56,58$ food 56,84

Thrysidium 532

thula, Egretta 152

Thyreoxenus 478

tibetanus, Ursus $26 \mathrm{ff}$

tibialis, Carcinops 617

ticks $579 \mathrm{ff}$

tiere, Holocentrus 92

Tinamus guttatus 12 major saturatus 9 osgoodi hershkovitzi 10

Tinodon 692

Titanosuchia $387,393,397$ origin 388

tolteca, Stenoderma 698

toltecus, Artibeus cinereus 698

torrei continentis, Chilonycteris 696

tortugae, Holocentrus 112

Toxochelyidae 365 plastron 377

Toxotes chatareus 52, 54, 55, 75

food 75,85

jaculator 74

jaculatrix 54, 74

food 74,85 
Traquairaspis 449

travassosi, Amblyopinodes 249

Tremarctos ornatus $25 \mathrm{ff}$

jaw mechanies 40

mandibular articulation 38

muscles 32

salivary glands 31

skull 26

tongue and oral vestibule 29

vessels and nerves 31

Tremarctotherium 25

Tretomys atavus 646

tri, Engraulis 56

Stolephorus 54, 56

food 57

Tribalinae 601

Tribalus 601

Trichiuridae 298

Trichopterygidae 561

Trichotermes 511

tridens, Asellia 595

Trinervitermes 473, 481, 486, 501, 514

Trinity Sand Formation 689

tripunctulatus, Valenciennellus 274

Trirhinopholis styani 174

Tristan da Cunha, land birds of $139 \mathrm{ff}$

trivittata, Beanea 95

troglodytes, Carcinops 605, 608, 618 Hister 618

Paromalus 618

tropica, Calamaria 168, 192, 206

truei gratus, Peromyscus 548

Tuberculitermes 494,511

tumens, Nenia (Neniatracta) adusta 305

Tumulitermes 469, 477, 503, 514

tupacii, Bulimulus 316

Turdus 144

turritus, Bulimulus 336

turtles, carettine $345 \mathrm{ff}$ loggerhead 345 ridley 345

Tylosurus strongylurus 53, 66 food 84

Typhlogeophis 168

typicus, Reithrodon 646

ulmeri, Calamaria 206

Ulmeriella 482, 490, 499, 507

umbilicatellus, Bulimulus infundibulum 320

umbilicatus, Platylomalus 619

umbratus, Vampyrops 408

undecimus, Subulitermes 502

undulatus, Crypturellus 16

Unguitermes 511

unicolor, Neopetraeus cora 335

uniformis, Calamaria 206

unimaculata, Betta 53, 54, 70

ecological distribution 82,86

food 70,84

Parophiocephalus 70 unipennis, Melanonus 291

Uralotermes 507

Uralotermitidae 506, 507

Urodela 531

urophthalmus, Eleotris 77

Oxyeleotris 52, 77 food 77,85

Urotriainus 561-563, 564, 567

bidentatus 563,564

grandis 564

robustior 564

molest $i 564$

ursinus, Melursus 25

Ursus americanus 25

tibetanus $26 \mathrm{ff}$

urubambensis, Nenia angrandi 306

urvillei, Hololepta 630

Platylister 630

Platysoma 605, 608, 630

Vaimosa bikolana 79

valdiviae, Notolychnus 288

Vale Formation 389

fauna $390 \mathrm{ff}$

Valenciennellus tripunctulatus 274

Vampyrops 403

dorsalis 407

fumosus 405

helleri 411

infuscus 405

lineatus 408, 409, 411

recifinus 413

umbratus 408

vittatus 405

zarhinus 411

vanum, Platysoma 631

variabilis, Periophthalmus 55, 79

Velocitermes 469, 496, 514

venezuelae, Holochilus 661, 669, 670 brasiliensis 670

venezuelensis, Nothocercus 14

venosus, Arius 65

Tachysurus 55, 65 food 84

ventralis, Calamaria 196, 206

vermiformis, Calamaria 176, 178, 183, 185, 207

vermiformis 187,207

grayi, Calamaria 207

Verrucositermes 515

vertebrae, of Caretta caretta 360

of Chelonia mydas 360

of Eretmochelys 360

of Lepidochelys 360

of Procolpochelys 360

Vertiginidae 305

veruculum, Bulimulus 320

vespertilionis, Argas 586, 596, 598

vestitus, Strophocheilus maximus 309

vexillarius, Holocentrus 92, 96, 100, 109

victoriae, Platylomalus 619

villai, Strepsylla 546 
Vinciguerria attenuata 273

nimbraria 273

poweriae 273

virgulata, Calamaria 180, 207

vittatus, Vampyrops 405

voeltzkowi, Psammotermes 488

vogti, Pteraspis 452

vulpinus, Hesperomys 657, 664

Holochilus 647, 657, 661, 663

Holochilus brasiliensis 661, 662

Mus 660

Sigmodon 657,668

warszewiczi, Nenia 307

waterhousei, Amblyopinus 219, 243

weiseri, Edrabius 255

weyrauchi, Nenia 308

whitei, Pteraspis 450

Wichita Group 389

fauna 390 wilkinsi, Nesospiza 147, 150

Winteriidae 284

xantherythrus, Holocentrus 92

yapura, Crypturellus undulatus 16

Yarella blakfordi 272

zamboangensis, Calamaria 207

zealandica, Sternaulax 626

Zenarchopterus amblyurus 52ff, 67 food 67,84

zilchi, Drymaeus 333

Zonibyx modestus 154

zoogeography, of New Caledonia Histeridae $605 \mathrm{ff}$

of termites $465 \mathrm{ff}$

Zootermopsis 489, 490, 507, 509

Zoothera 144 







UNIVERSITY OF ILLINOIS-URBANA

$590.5 \mathrm{FI}$

FIELDIANA, ZOOL OGYSCHGO

37-38 1955-56

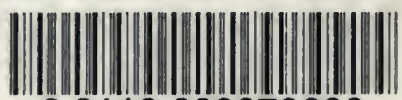

30112009379683 